\title{
SCHEDULING IMPATIENT JOBS IN A CLEARING SYSTEM WITH INSIGHTS ON PATIENT TRIAGE IN MASS CASUALTY INCIDENTS
}

\author{
Nilay Tanık Argon*, Serhan Ziya*, Rhonda Righter** \\ *Department of Statistics and Operations Research, University of North Carolina, Smith \\ Building CB\#3260, Chapel Hill, NC 27599-3180, U.S.A. \\ **Department of Industrial Engineering and Operations Research, University of California, \\ Berkeley, CA 94720-1777, U.S.A.
}

October 12,2007

\begin{abstract}
Motivated by the patient triage problem in emergency response, we consider a single-server clearing system in which jobs may abandon the system if they are not taken into service within their "lifetime." In this system, jobs are characterized by their lifetime and service time distributions. Our objective is to dynamically determine the optimal or near-optimal order of service for jobs so as to minimize the total number of abandonments. We first show that if the jobs can be ordered in such a way that the job with the shortest lifetime (in the sense of hazard rate ordering) also has the shortest service time (in the sense of likelihood ratio ordering), then the optimal policy gives the highest priority to this "time-critical" job independently of the system state. For the case where the jobs with shorter lifetimes have longer service times, we observed that the optimal policy generally has a complex structure that may depend on the type and number of jobs available. For this case, we provide partial characterizations of the optimal policy and obtain sufficient conditions under which a state-independent policy is optimal. Furthermore, we develop two state-dependent heuristic policies, and by means of a numerical study, show that these heuristics perform well, especially when jobs abandon the system at a relatively faster rate when compared to service rates. Based on our analytical and numerical results, we develop several insights on patient triage in the immediate aftermath of a mass casualty event. For example, we conclude that in a worstcase scenario, where medical resources are overwhelmed with a large number of casualties who need immediate attention, it is crucial to implement state-dependent policies such as the heuristic policies proposed in this paper.
\end{abstract}

Keywords: Priority scheduling, stochastic orders, stochastic dynamic programming, impatient customers, abandonment, reneging, patient triage, emergency response. 


\section{Introduction}

Consider a service system where a finite number of jobs are waiting to receive service from a single server. There will be no additional arrivals to this system and the existing jobs may abandon the system without receiving service if they are not given service within their lifetime. For such a system, we are interested in the dynamic scheduling of jobs that are characterized by their lifetime and service time distributions with the objective of minimizing the expected number of total abandonments. More specifically, our aim is to develop insights on optimal or near-optimal scheduling policies that determine the priorities among different job types dynamically depending on the system state.

Our primary motivation to study this optimal control problem originates from the patient triage ${ }^{1}$ problem that arises in the immediate aftermath of a mass casualty incident such as a natural disaster or a terrorist attack. After such an event, which may cause a significant number of injuries and overwhelm the local medical resources, emergency responders perform triage on patients and determine the order by which these patients will be admitted to scarce resources. These resources may include ambulances, imaging devices (e.g., X-ray and MRI machines), or operating rooms. For example, in case of a mass trauma event caused by a bombing, it is estimated that at least half of the casualties will require surgical procedures, and therefore operating rooms are expected to constitute a serious bottleneck (see, e.g., Levi et al. [18] and Peleg et al. [21]).

Many local emergency response divisions and hospitals have adopted triage procedures that will be used in case of a mass casualty incident. Following these procedures, immediately after the incident, patients are classified into different priority groups. (According to one of the most widely adopted triage procedures, which is called START, patients are classified into four groups: immediate, delayed, minimum, and expectant; see, e.g., Nocera and Garner [19].) Then, each patient is given treatment in the order determined by his/her priority class. According to these triage procedures, patients are typically classified into priority classes based solely on their initial health conditions, ignoring other factors such as the size of the event (i.e., the number of patients waiting for care) and treatment times. Furthermore, these initially assigned priorities do not change with any changes in the system state, i.e., the number of patients at different criticality levels and time. However, recent work from the emergency response literature suggests that when determining priorities, the numbers of patients at different criticality levels should also be considered to achieve the greatest good for the greatest number (see Arnold et al. [1] and Frykberg [12]). Indeed, one of the findings

\footnotetext{
${ }^{1}$ Triage is a brief clinical assessment that determines the order in which patients should be seen in the Emergency Department or, if in the field, the speed of transport and choice of hospital destination [26].
} 
of our study is that making prioritization decisions dynamically over time, based on the system state, can bring significant improvements in the number of patients saved.

We first investigate conditions under which one can safely ignore the system state (i.e., the composition of patients at different criticality levels and time) in making prioritization decisions. For example, we find that if the patient with the shortest service time (in the sense of likelihood ratio ordering) also has the shortest lifetime (in the sense of hazard rate ordering), then priority should be given to this patient independently of the system state. However, in general, we show that there are clear benefits of updating priorities as the system state changes. We prove structural results for our optimal control problem, and propose easy-to-implement and insightful heuristic policies that will help make these dynamic prioritization decisions. Our numerical experiments show that these heuristic policies perform quite well.

The outline of the paper is as follows. In Section 2, we provide a literature review on scheduling, queueing, and optimal control problems that are most relevant to our problem. In Section 3, we formally define the optimal control problem under consideration, and obtain analytical results on the characteristics of this problem and its solution for general service time and lifetime distributions. In Section 4, we consider the Markovian case where the service times and lifetimes are exponentially distributed. Under certain conditions on the service and abandonment rates, we provide a complete characterization of the optimal policy for the Markovian case. Based on our analytical results, we propose two heuristic policies that are described in Section 5. A numerical study that tests the performance of these policies is presented in Section 6. Finally, Section 7 includes our concluding remarks and discussions.

Before we proceed further, we would like to mention two important points regarding this paper. First, it should be noted that patient triage is an extremely complicated decision problem that involves a significant degree of human judgment in classifying patients and determining priorities. Any mathematical model of this problem that is also analytically tractable needs to be a significantly simplified version of the reality. Therefore, our objective here is not to develop policies that can be readily implemented but rather to provide some general insights on the problem. Second, although our main focus in this paper is on the patient prioritization problem, our treatment of the problem is general enough to be relevant to other application areas as well (see Glazebrook et al. [14] for examples of other applications). Hence, in order to keep the general appeal of our results, throughout the paper, we use the general queueing terminology, e.g., by referring to patients as "jobs" and operating times as "service times." However, we discuss most of our results and observations in relation to the patient triage problem when they are especially relevant and significant within that context. 


\section{Literature review and contribution of the paper}

Although the problem under consideration has relevance to several different areas in the literature, to our knowledge, the only closely related study is conducted by Glazebrook, Ansell, Dunn, and Lumley [14]. In this section, we first review the paper by Glazebrook et al. [14], and then provide a survey of the relevant literature on stochastic scheduling and optimal control of queueing and clearing systems with reneging (abandonment).

Glazebrook et al. [14] considers scheduling of jobs in a clearing system with impatient jobs that abandon the system unless they receive service before their random due dates. One of the models studied in Glazebrook et al. [14] is concerned with the scheduling of jobs in a clearing system with $N \geq 2$ jobs having exponentially distributed times to abandonment, where jobs are characterized by their abandonment rates, mean service times, and rewards. The objective is to maximize the total expected reward earned. The authors prove that a policy resembling the " $c \mu$ rule" is asymptotically optimal in the class of non-preemptive policies as the abandonment rates approach zero. The authors also provide numerical results on the performance of the suggested policy. To our knowledge, this heuristic method is the only other policy available in the literature that is alternative to those that we provide in this study. We discuss the policy by Glazebrook et al. [14] in more detail in Section 5, and compare its performance with the performance of our heuristics by means of a numerical study in Section 6.

There are several other papers in the general context of scheduling in queueing systems with random or predetermined deadlines for jobs, and queueing systems with expulsion (where the system controller may eject jobs), see, e.g., Bhattacharya and Ephremides [3, 4], Doytchinov, Lehoczky, and Shreve [10], Glazebrook [13], Jang and Klein [16], Jiang, Lewis, and Colin [17], Righter [25], Panwar, Towsley, and Wolf [20], Van Mieghem [28], $\mathrm{Xu}$ [33], and Zhao, Panwar, and Towsley [34]. Among these papers, we find Bhattacharya and Ephremides [3, 4], Panwar et al. [20], and Zhao et al. [34] to be the most relevant to our work mainly because the performance measure of interest in these papers is the (weighted) number of tardy jobs, i.e., jobs whose deadline expires while waiting in the queue. Bhattacharya and Ephremides [4] and Panwar et al. [20] study the scheduling problem under the assumption that the stochastic due date of a job is announced upon the arrival of the job, and show that a form of the "shortest-time-to-extinction" policy is optimal under certain conditions. Bhattacharya and Ephremides [3] and Zhao et al. [34], on the other hand, assume that the decision maker knows only the distribution of the due date of a job, not the exact due dates, at any decision epoch. In particular, Bhattacharya and Ephremides [3] show that under the assumption of independent and identically distributed (i.i.d.) lifetimes, i.i.d. service times, 
and i.i.d. interarrival times (that are all mutually independent), the "earliest-arrival" policy is optimal if the lifetime distribution has a non-decreasing failure rate.

There are also several papers on the scheduling of jobs in (stochastic) clearing systems, see, e.g., Boxma and Forst [5], Coffman, Flatto, Garey, and Weber [9], Emmons and Pinedo [11], Pinedo [22], Righter [24], Weber, Varaiya, and Walrand [31], and Weiss and Pinedo [32]. Among these papers, Boxma and Forst [5], Emmons and Pinedo [11], and Pinedo [22] are the most relevant to our work since they focus on the objective of minimizing the (weighted) number of tardy jobs. In these three papers, all jobs are available at time zero (except in Pinedo [22]), they have job-dependent stochastic due dates, and processing times are stochastic. The objective is to obtain a job sequence that minimizes the mean number of tardy jobs (i.e., jobs that are not completed by their due date). Two types of policies were considered in these papers: (static) list scheduling policies and dynamic policies. Under list scheduling policies, the decision maker arranges all jobs into a list at time zero, and is not allowed to change this list thereafter. Hence, when a list scheduling policy is applied, all jobs (even those jobs that are tardy) are processed. Under dynamic policies, on the other hand, the decision maker is allowed to modify earlier decisions at any time as the new information becomes available. Pinedo [22] and Boxma and Forst [5] consider only list scheduling policies, whereas Emmons and Pinedo [11] consider both list scheduling and dynamic policies. In particular, Pinedo [22] shows that if the processing times of jobs are independent and exponentially distributed, their release dates (i.e., the times that the jobs are available for processing) are random, and their due dates are identically distributed, then the optimal static list policy sequences jobs in increasing order of mean processing times when the system has a single server. Boxma and Forst [5] study the same problem (except that all jobs are available for processing at time zero) and identify optimal static list policies under several sets of conditions on due date and processing time distributions. One of the results proved by Boxma and Forst [5] states that if all due dates are i.i.d. and a stochastic ordering exists among the processing time distributions, then the jobs should be sequenced according to the increasing stochastic ordering, i.e., jobs with stochastically shortest processing times should be processed first. Emmons and Pinedo [11] study the same problem but with multiple servers. They provide a set of special cases for which optimal dynamic policies or list policies can be identified. One of their results states that if the processing times are i.i.d. exponential, and the due dates are independent and can be ordered according to their failure rates, then the optimal preemptive dynamic policy is to process the jobs in the increasing order of their failure rates.

Although there is a clear connection between our work and the stochastic scheduling literature reviewed above, none of these papers considers the problem studied in this pa- 
per. Almost all of the stochastic scheduling problems for clearing systems with an objective of minimization of number of tardy jobs focus on (static) list policies in contrast to dynamic policies that we consider in this paper. In our model, jobs abandon the system once their "deadline" is reached, and thus they cannot be processed afterwards. Because of this property, the system state (i.e., the set of jobs in the system) changes after every job abandonment. Therefore, there are potential benefits of assigning priorities dynamically in time. Indeed, we provide several examples in the paper to show that policies that are not dynamic in nature may perform very poorly in our case. Furthermore, the majority of papers on stochastic scheduling aim at identifying conditions that are generally in the form of an ordering condition among lifetimes and service times, under which a list scheduling policy is optimal. These conditions typically require the service times and lifetimes of jobs to be agreeably ordered (i.e., jobs with shorter lifetimes to also have shorter service times), which does not hold in many practical settings. In the first part of the paper, we identify conditions under which state-independent policies are optimal. However, the main focus of the paper is on obtaining good policies for all possible situations (especially when the service times and lifetimes are not agreeably ordered). Therefore, a significant portion of this paper deals with obtaining near-optimal policies that would work well under all possible conditions.

Finally, we note that our model in general can be viewed as a queueing system with reneging (abandonment), and there is a vast literature on this topic. For some recent work on queueing systems with reneging, see, e.g., Bae, Kim, and Lee [2], Brandt and Brandt [6, 7], Choi, Kim, and Chung [8], Ward and Glynn [29], and Ward and Kumar [30].

\section{General service time and lifetime distributions}

In this paper, we consider a single server clearing system initially having $N \geq 2$ jobs that may abandon the system before they receive service. Throughout the paper, we refer to the maximum time a job can tolerate waiting in the queue as the lifetime of the job. If the lifetime of a job expires before it is taken into service, then the job abandons the system. We assume that a job that is already taken into service does not abandon the system. We also assume that the service is performed in a non-preemptive manner, i.e., once the server starts processing a job, it cannot start working on another job before completing the processing of the job that is already in service. This assumption is reasonable within the context of the patient triage problem, since it is generally not desirable to interrupt a medical procedure.

We let $Y_{i}$ be the random variable representing the lifetime of job $i$ at time zero, and $S_{i}$ be the random variable representing the service time of job $i$ for $i=1,2, \ldots, N$. We also define $C_{\pi}(t)$ to be the total number of jobs taken into service by time $t \geq 0$ when policy 
$\pi$ is applied, where $\pi \in \Pi$ and $\Pi$ is the set of all admissible, dynamic, and non-preemptive scheduling (prioritization) policies. A dynamic prioritization policy is a collection of rules that determines which job the server takes into service at any given decision epoch based on the state of the system (i.e., the time of the decision epoch and the set of jobs in the system). (As we show in Proposition 1 below, idling is suboptimal, and hence decision epochs are the time instances when service completions occur.) Our objective is to identify characteristics of policies that maximize $C_{\pi}(t)$ stochastically, and hence maximize the expected total number of jobs served when the system is cleared.

In the remainder of this section, we provide characterizations of the optimal control problem described above without making any distributional assumptions for service times and lifetimes. First, note that a standard coupling argument can be applied to prove that an idling policy (i.e., a policy under which the server may idle in the presence of jobs) can never be optimal.

Proposition 1 Any idling policy is suboptimal in the sense of maximizing $C_{\pi}(t)$ along any given sample path.

Based on Proposition 1, in the rest of the paper, we only consider non-idling policies. We next provide a complete characterization of the optimal policy when service times and lifetimes of jobs are "agreeably ordered" according to certain stochastic orders. We first provide definitions of three stochastic orders. Suppose that $X$ and $Y$ are two random variables. If $\operatorname{Pr}\{X>u\} \leq \operatorname{Pr}\{Y>u\}$, for all $u \in(-\infty, \infty)$, then $X$ is said to be smaller than $Y$ in the sense of usual stochastic orders (denoted by $X \leq_{s t} Y$ ). On the other hand, if $\operatorname{Pr}\{X-v>u \mid X>v\} \leq \operatorname{Pr}\{Y-v>u \mid Y>v\}$, for all $u \geq 0$ and $v \in(-\infty, \infty)$, then $X$ is said to be smaller than $Y$ in the sense of hazard rate orders (denoted by $X \leq{ }_{h r} Y$ ). Finally, let $f(t)$ and $g(t)$ be the densities or probability mass functions of $X$ and $Y$, respectively. If $f(t) / g(t)$ is decreasing in $t$ over the union of the supports of $X$ and $Y$, then $X$ is said to be smaller than $Y$ in the sense of likelihood ratio orders (denoted by $X \leq_{l r} Y$ ). We will need the following lemma (see, e.g., Lemma 13.D.1 in Righter [23]) to prove Theorem 1.

Lemma 1 Let $X$ and $Y$ be two independent random variables. Then, $X \leq_{l r} Y$ if and only if $(X \mid \min (X, Y)=m, \max (X, Y)=M) \leq_{s t}(Y \mid \min (X, Y)=m, \max (X, Y)=M)$ for all $m \leq M$.

In other words, given $m=\min (X, Y)$ and $M=\max (X, Y)$, we have that $X \leq_{l r} Y$ if and only if $P\{X=m \mid m, M\}=P\{Y=M \mid m, M\} \geq P\{X=M \mid m, M\}=P\{Y=m \mid m, M\}$. Note that $X \leq_{l r} Y \Rightarrow X \leq_{h r} Y \Rightarrow X \leq_{s t} Y$. 
Theorem 1 If $Y_{1} \leq_{h r} Y_{2} \leq_{h r} \cdots \leq_{h r} Y_{N}$ and $S_{1} \leq_{l r} S_{2} \leq_{l r} \cdots \leq_{l r} S_{N}$, then a nonpreemptive and non-idling policy that prioritizes the job with the smallest index at any decision epoch maximizes $\left\{C_{\pi}(t)\right\}_{t=0}^{\infty}$ in the sense of usual stochastic orders.

Proof: We use induction on the number of jobs, so suppose the theorem is true when there are $k \leq N-1$ jobs and consider the case of $N$ jobs. (Note that the theorem holds trivially when $N=1$.) Suppose policy $\pi$ does not comply with the smallest index (SI) policy given in Theorem 1 at time 0 , i.e., policy $\pi$ takes job $j$ into service at time 0 , where $j \in\{2,3, \ldots, N\}$. We will construct a policy $\gamma$, which serves job 1 at time 0 , and for which $C_{\pi}(t) \leq C_{\gamma}(t)$ for all $t \geq 0$ along any given sample path. The SI policy, which agrees with $\gamma$ for the first decision and is optimal thereafter from the induction hypothesis, will then have $C_{\mathrm{SI}}(t) \geq C_{\gamma}(t)$ for all $t \geq 0$.

First suppose $\pi$ does not agree with the SI policy for some later decisions, as well as the decision at time 0 . Then, from the induction hypothesis, we can let $\gamma$ serve job $j$ at time 0 and then agree with the SI policy for all later decisions, such that $C_{\pi}(t) \leq C_{\gamma}(t)$ for all $t \geq 0$. Therefore, without loss of generality, assume $\pi$ agrees with the SI policy after the first decision, and in particular, that $\pi$ serves job 1 after job $j$, if job 1 is still available. Let $\gamma$ serve job $j$ after job 1, if job $j$ is still available, and let it agree with $\pi$ (and the SI policy) thereafter. Let $Y_{i}^{\rho}, \rho=\pi, \gamma$, denote the remaining lifetime of job $i$ at time 0 under policy $\rho$, where $i=1,2, \ldots, k$. Note that by the stochastic ordering relation among remaining lifetimes of jobs, we can couple the random variables so that $Y_{1}^{\pi}=y_{1} \leq y_{j}=Y_{j}^{\gamma}$. Because policy $\pi(\gamma)$ serves job $j(1)$ at time 0 , and the job that is in service will not abandon, we do not need $Y_{j}^{\pi}$ or $Y_{1}^{\gamma}$. Let $Y_{i}^{\gamma}=Y_{i}^{\pi}$ for all $i \neq 1, j$. Let $S_{i}^{\rho}, \rho=\pi, \gamma$, denote the service time of job $i$ under policy $\rho$, and let $S_{i}^{\gamma}=S_{i}^{\pi}$ for all $i \neq 1, j$. From Lemma 1, we can couple $\left(S_{1}^{\pi}, S_{j}^{\pi}\right)$ with $\left(S_{1}^{\gamma}, S_{j}^{\gamma}\right)$ so that $m=\min \left\{S_{1}^{\pi}, S_{j}^{\pi}\right\}=\min \left\{S_{1}^{\gamma}, S_{j}^{\gamma}\right\} \leq M=\max \left\{S_{1}^{\pi}, S_{j}^{\pi}\right\}=$ $\max \left\{S_{1}^{\gamma}, S_{j}^{\gamma}\right\}$ and either $S_{j}^{\pi}=S_{1}^{\gamma}=: a \in\{m, M\}$ and $S_{1}^{\pi}=S_{j}^{\gamma}=: b \in\{m, M\} \backslash\{a\}$ (Case I) or $S_{1}^{\pi}=S_{1}^{\gamma}=m \leq S_{j}^{\pi}=S_{j}^{\gamma}=M($ Case II).

Case Ia: We first consider the case where $a<y_{1}$. Then $\pi$ will serve job 1 at time $a$, and $\gamma$ will serve job $j$. From time $a+b$ on, the states will be the same under both policies, and we have $C_{\gamma}(t)=C_{\pi}(t)$ for all $t \geq 0$.

Case Ib: Now suppose $y_{1} \leq a<y_{j}$. Let $C^{\prime}(t), t \geq a$, be the number of jobs taken into service under the SI policy starting from time $a$ and assuming the first job completion occurs at time $a$, and with the state the same as under $\pi$ at time $a$, and with all random variables coupled to be the same as those under $\pi$, except that we assume job 1 is still present at time $a$, with remaining life from time $a$ of $Y_{1}(a)={ }_{s t}\left(Y_{1}-a \mid Y_{1}>a\right)$. Let $\pi^{\prime}$ be the corresponding policy. Then, arguing as in Case Ia, $C_{\gamma}(t)=C^{\prime}(t)$ for $t \geq a$, and $C_{\gamma}(t)=C_{\pi}(t)$ for $0 \leq t \leq a$. 
It remains to show that $C^{\prime}(t) \geq C_{\pi}(t)$ for $t \geq a$. Define a new policy $\pi^{\prime \prime}$ that starts in the same state as $\pi^{\prime}$ at time $a$, and that follows the SI policy except that it serves job 1 last (at time $\tau$ say), if it is still available. Then, for $a \leq t \leq \tau, C_{\pi^{\prime \prime}}(t)=C_{\pi}(t)$, and for $t \geq \tau, C_{\pi^{\prime \prime}}(t) \geq C_{\pi}(t)$. Since $\pi^{\prime}$ agrees with the SI policy, from the induction hypothesis, $C^{\prime}(t) \geq C_{\pi^{\prime \prime}}(t)$ for all $t \geq a$.

Case Ic: Suppose $y_{j} \leq a$. Then from time $a$ on the states will be the same under both policies, and we have $C_{\gamma}(t)=C_{\pi}(t)$ for all $t \geq 0$.

Case IIa: Suppose $M<y_{1}$, and therefore $m<y_{j}$. At time $m+M$ the states under $\pi$ and $\gamma$ are the same, so $C_{\gamma}(t)=C_{\pi}(t)$ for $t \geq m+M$. Before time $m+M$, we have $C_{\gamma}(t)=C_{\pi}(t)=1$ for $0 \leq t<m, C_{\gamma}(t)=2>C_{\pi}(t)=1$ for $m \leq t<M$, and $C_{\gamma}(t)=C_{\pi}(t)=2$ for $M \leq t<m+M$. Thus, we have $C_{\gamma}(t) \geq C_{\pi}(t)$ for all $t \geq 0$.

Case IIb: Suppose $m<y_{j}$ and $y_{1} \leq M$. Then we can argue as in Case Ib that $C_{\gamma}(t) \geq C_{\pi}(t)$, $t \geq 0$.

Case IIc: We finally consider the case where $y_{j} \leq m$, and therefore, $y_{1}<M$. Let $C^{\prime}(t)$ be the number of jobs taken into service by time $t$ under the SI policy starting from time $m$ with the state the same as under $\pi$ at time $m$, and with all random variables coupled to be the same as those under $\pi$, except that we assume job $j$ finishes at time $m$ instead of time $M$, and let $\pi^{\prime}$ be the corresponding policy. Then $C^{\prime}(t)=C_{\gamma}(t)$ for $t \geq m$, and $C_{\gamma}(t)=C_{\pi}(t)$, $0 \leq t<m$, so we need to only show that $C^{\prime}(t) \geq C_{\pi}(t)$ for $t \geq m$. Note that $C_{\pi}(t), t \geq m$, is the same as if we started with the same state as $\pi^{\prime}$ but idled from time $m$ to $M$ and then followed the SI policy, and did not count the job completion until time $M$, so $C^{\prime}(t) \geq C_{\pi}(t)$ for $t \geq m$ from Proposition 1 and the induction hypothesis.

Theorem 1 implies that if jobs can be ordered in such a way that the one with the shortest lifetime in the sense of hazard rate orders also has the shortest service time in the sense of likelihood ratio orders, then giving this job priority for service maximizes the expected total number of jobs served. We next provide a definition that we will frequently use in the remainder of the paper.

Definition 1 If $Y_{i} \leq_{h r} Y_{j}$, then job $i$ is said to be more time-critical than job $j$, for $i, j \in$ $\{1,2, \ldots, N\}$ and $i \neq j$.

Using this definition, Theorem 1 states that regardless of the system state, time-critical jobs with shorter service times in the sense of likelihood ratio orders should always be given priority for service. Theorem 1 provides us with a criterion as to what makes a job a toppriority job. This is especially important in the context of patient triage, since it implies that 
a patient with a certain injury can be given the highest priority irrespective of the number of other patients if his/her lifetime and service time are shorter than those for any other patient in the sense of hazard rate and likelihood ratio orders, respectively.

Remark 1 In certain applications, serving different jobs may not bring the same amount of benefit. For example, in the context of patient triage, certain operations may be riskier than others in that the chance of survival of a patient after such an operation may be lower than that for other operations. In such a situation, instead of maximizing the number of patients taken into service, it is more sensible to maximize the average number of patients saved by taking into account risk factors associated with the operation of each patient. To formulate such an objective function, let $\theta_{i}$ be the fixed reward earned by serving job $i$, for $i=1,2, \ldots, N$, and let $C_{\pi}(t)$ be the total reward earned by time $t$ under policy $\pi$. (For the patient triage problem, $\theta_{i}$ may denote the probability of survival of patient $i$ after he/she is taken into operation.) Proposition 1 trivially holds under this new performance measure. Furthermore, we can also show that Theorem 1 holds if in addition to the stochastic ordering conditions on the service time and lifetimes given in Theorem 1, we also have $\theta_{1} \geq \theta_{2} \geq \cdots \geq \theta_{N}$. In other words, if serving jobs with stochastically smaller service times and lifetimes brings larger rewards, then these jobs should be given higher priority no matter what the state of the system is.

In most applications, it may not be practically feasible to characterize each job in the system with its own lifetime and service distribution. For example, for the patient triage problem, it is common practice to classify patients into at most three or four categories. Especially when there is a time pressure as in the case of mass casualty events, patients' conditions are quickly assessed right after the event, and then they are classified into a small number of priority classes even though each patient has his/her own unique injuries. Based on this, in the remainder of the paper, we will consider the case where jobs are classified into only two types, each type having its own lifetime and service time distribution. Let $m_{i}$ be the number of type $i \in\{1,2\}$ jobs initially in the system such that $m_{1}+m_{2}=N$.

For the problem with two types of jobs in the system, Theorem 1 implies that a job type with shorter lifetimes (in the sense of hazard rate orders) and shorter service times (in the sense of likelihood ratio orders) should always receive priority for service no matter how many jobs from each type are present in the system. Although this result provides us a criterion as to what makes a type a top-priority class, a more interesting (perhaps a more common) case is when time-critical jobs have longer service times. In Sections 4, 5, and 6, our main focus will be on this case. 


\section{Exponential service time and lifetime distributions}

In this section, we study the same model described in Section 3 except that we now assume that jobs are categorized into two classes and that their service times and lifetimes are exponentially distributed. For $i=1,2$, let $\mu_{i}>0$ and $r_{i}>0$ be the service rate and lifetime rate for a type $i$ job, respectively. Let also $D_{\pi}\left(m_{1}, m_{2}\right)$ denote the expected total number of jobs taken into service when the system is cleared if scheduling (prioritization) policy $\pi \in \Pi$ is applied and $m_{i}$ jobs of type $i \in\{1,2\}$ are initially in the system. We use a dynamic programming formulation to find an optimal or near-optimal solution to the optimization problem stated as

$$
\max _{\pi \in \Pi} D_{\pi}\left(m_{1}, m_{2}\right) \text {. }
$$

We define the state of the system as $\left(x_{1}, x_{2} ; Q\right)$, where $x_{i}$ is the number of type $i$ jobs in the system and $Q \in\left\{P_{1}, P_{2}, R\right\}$ is the status of the server. When $Q=P_{i}$ it means that the server is processing a job of type $i \in\{1,2\}$, and when $Q=R$ it means that the server is ready to process a new job. Let $V\left(x_{1}, x_{2} ; Q\right)$ be the maximum expected number of jobs served starting from state $\left(x_{1}, x_{2} ; Q\right)$. Then, using the convention that $V\left(0, x_{2} ; P_{1}\right)=V\left(x_{1}, 0 ; P_{2}\right)=0$, where $x_{i}=0,1, \ldots, m_{i}$ for $i=1,2$ and the notation that $\mathbb{I}_{A}$ is the indicator function of event $A$, the dynamic programming equations are given as follows:

$$
\begin{gathered}
V\left(x_{1}, x_{2} ; R\right)=\mathbb{I}_{\left\{x_{1}+x_{2} \geq 1\right\}}+\max \left\{V\left(x_{1}, x_{2} ; P_{1}\right), V\left(x_{1}, x_{2} ; P_{2}\right)\right\}, \forall x_{i}=0,1, \ldots, m_{i}, i=1,2, \\
V\left(x_{1}, x_{2} ; P_{1}\right)=\frac{\mu_{1} V\left(x_{1}-1, x_{2} ; R\right)+\left(x_{1}-1\right) r_{1} V\left(x_{1}-1, x_{2} ; P_{1}\right)+x_{2} r_{2} V\left(x_{1}, x_{2}-1 ; P_{1}\right)}{\mu_{1}+\left(x_{1}-1\right) r_{1}+x_{2} r_{2}}, \\
\forall x_{1}=1,2, \ldots, m_{1} \text { and } x_{2}=0,1, \ldots, m_{2} ; \\
V\left(x_{1}, x_{2} ; P_{2}\right)=\frac{\mu_{2} V\left(x_{1}, x_{2}-1 ; R\right)+x_{1} r_{1} V\left(x_{1}-1, x_{2} ; P_{2}\right)+\left(x_{2}-1\right) r_{2} V\left(x_{1}, x_{2}-1 ; P_{2}\right)}{\mu_{2}+x_{1} r_{1}+\left(x_{2}-1\right) r_{2}}, \\
\forall x_{1}=0,1, \ldots, m_{1} \text { and } x_{2}=1,2, \ldots, m_{2} .
\end{gathered}
$$

We will use this dynamic programming formulation to identify optimal or near-optimal dynamic prioritization policies that maximize the expected total number of jobs served. First, note that Theorem 1 implies that for this Markovian system if type 1 jobs have shorter mean service times and lifetimes than type 2 jobs, then type 1 jobs should be given priority for service regardless of the system state. This is in agreement with one's intuition since there seems to be no reason to give priority to less time-critical jobs when serving them takes at least as much time as serving more time-critical jobs. Thus, the more interesting (and realistic) case is when time-critical jobs have longer mean service times, i.e., $\mu_{1}<\mu_{2}$ and $r_{1}>r_{2}$. We focus on this case in the remainder of this section.

From our numerical experiments, we observed that the optimal policy gives higher priority to jobs that are less time-critical and that have shorter service times, when the number 
of jobs from each type is sufficiently large. As the number of jobs from each type drops, giving priority to more time-critical jobs that require longer service times becomes a better strategy. Figure 1 demonstrates this behavior with $\mu_{1}<\mu_{2}$ and $r_{1}>r_{2}$. In the context of emergency response planning, this suggests that depending on the number of patients at different criticality levels it might be better to give priority to less time-critical patients. This contradicts the general belief and the common practice that gives priority to time-critical patients at all times, and it strongly supports the argument that when the number of casualties is high, emergency resources should be allocated to less time-critical patients with shorter treatment times as the objective is to do the greatest good for the greatest number. (Indeed, as $m_{1} \rightarrow \infty$ and $m_{2} \rightarrow \infty$, our objective essentially becomes to maximize the throughput, and hence shortest-expected-processing-time-first rule (SEPT) becomes the optimal policy.) In order to implement this principle, one needs to first answer the following question: What number of jobs is considered to be sufficiently high to follow this principle? In the remainder of this section, we present some results aiming at answering this question.

Figure 1: A typical structure for the optimal policy when $\mu_{1}<\mu_{2}$ and $r_{1}>r_{2}$.

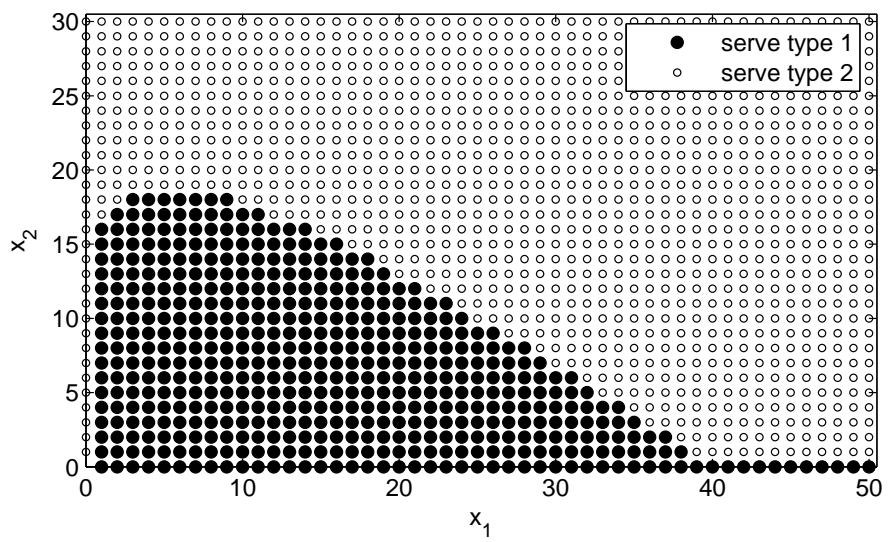

We first present a proposition that gives a necessary condition for an index policy to be optimal. An index policy is a state-independent policy that gives priority based only on job type, or index, at any state $\left(x_{1}, x_{2}\right)$. For example, SEPT is an index policy that always gives priority to the job with the largest value of $\mu$. Similarly, the $r \mu$ rule of Proposition 2 is the index policy that always gives priority to the job with the largest value of $r \mu$. Note that the $r \mu$ rule resembles the well-known $c \mu$ rule in queueing theory since it gives priority to job type $i$ with the highest value of $r_{i} \mu_{i}$. (For a comprehensive literature review on the $c \mu$ rule, the interested reader is referred to Van Mieghem [27].) 
Proposition 2 If there is an optimal policy among the set of index policies, it must agree with the $r \mu$ rule.

Proof: It is easy to check that $V\left(1,1 ; P_{1}\right) \geq V\left(1,1 ; P_{2}\right)$ if and only if $r_{1} \mu_{1} \geq r_{2} \mu_{2}$.

Note that Theorem 1 is consistent with Proposition 2 because $r_{1} \geq r_{2}$ and $\mu_{1} \geq \mu_{2}$, which implies that $r_{1} \mu_{1} \geq r_{2} \mu_{2}$. We next present four results that will be used in identifying optimal or near-optimal policies for the case when $\mu_{1}<\mu_{2}$ and $r_{1}>r_{2}$. More specifically, Proposition 3, which is a rather technical result, will be instrumental in the development of two heuristic policies as we explain in Section 5. Proposition 3 is also used in proving Propositions 4, 5, and 6, which provide sufficient conditions under which the optimal policy can be characterized.

In the proof of Proposition 3, we use the following lemma, which states that for a fixed number of type 1 and type 2 jobs, $x_{1}$ and $x_{2}$ in queue, we prefer to have the job in service be a job with a smaller mean service time. This makes sense because the remaining lifetime of the job in service is no longer relevant.

Lemma 2 If $\mu_{1} \leq \mu_{2}$, then we have $V\left(x_{1}+1, x_{2} ; P_{1}\right) \leq V\left(x_{1}, x_{2}+1 ; P_{2}\right)$ for all $x_{1}=$ $0,1, \ldots, m_{1}$ and $x_{2}=0,1, \ldots, m_{2}$.

Proof: We can couple the processing times of the jobs in service for the two states such that $S_{2} \leq S_{1}$ with probability one, where $S_{i}$ denotes the processing time of a type $i$ job. Let $V_{0}\left(x_{1}, x_{2}+1 ; P_{2}\right)$ be the value function when the starting state is $\left(x_{1}, x_{2}+1 ; P_{2}\right)$ and we idle from time $S_{2}$ to $S_{1}$ and then follow the optimal policy. Then, from Proposition 1, we have $V\left(x_{1}, x_{2}+1 ; P_{2}\right) \geq V_{0}\left(x_{1}, x_{2}+1 ; P_{2}\right)=V\left(x_{1}+1, x_{2} ; P_{1}\right)$.

Remark 2 Note that in the proof of Lemma 2, we have actually shown a stronger result. Suppose we have an arbitrary number of types of jobs, with arbitrary lifetime and service time distributions, and let $V_{t}\left(\mathbf{x} ; P_{i}\right)$ be the value function when the numbers of each type of job are given by the vector $\mathbf{x}$, the current time is $t$, and a job of type $i$ starts service at time $t$. Let also $\mathbf{e}_{\mathbf{i}}$ be a vector with a one in the $i$ th position and zeroes elsewhere. Then, we have shown that if $S_{i} \geq_{s t} S_{j}$, then $V_{t}\left(\mathbf{x}+\mathbf{e}_{\mathbf{i}} ; P_{i}\right) \leq V_{t}\left(\mathbf{x}+\mathbf{e}_{\mathbf{j}} ; P_{j}\right)$.

We are now ready to prove Proposition 3. Proposition 3 gives us conditions for the monotonicity of the policy in the state. To be more specific, it gives us conditions such that if it is optimal to serve a type $i$ job in state $\left(x_{1}, x_{2}-1\right)$ and $\left(x_{1}-1, x_{2}\right)$, then it is also optimal to serve a type $i$ job in state $\left(x_{1}, x_{2}\right)$. Note that these conditions require that the preferred job agrees with the $r \mu$ rule. 
Proposition 3 (i) Suppose $\mu_{1}<\mu_{2} \leq r_{2}, \mu_{1} \leq r_{1}$ and fix $x_{1} \geq 1, x_{2} \geq 1$. If

$$
x_{1} r_{1}+x_{2} r_{2} \geq \frac{r_{1} \mu_{2}-r_{2} \mu_{1}}{\mu_{2}-\mu_{1}}
$$

and if $V\left(x_{1}-1, x_{2} ; P_{2}\right) \geq V\left(x_{1}-1, x_{2} ; P_{1}\right)$, and, for $x_{2} \neq 1$, if $V\left(x_{1}, x_{2}-1 ; P_{2}\right) \geq V\left(x_{1}, x_{2}-\right.$ $\left.1 ; P_{1}\right)$, then $V\left(x_{1}, x_{2} ; P_{2}\right) \geq V\left(x_{1}, x_{2} ; P_{1}\right)$.

(ii) Suppose $r_{1} \leq \mu_{1}<\mu_{2}, r_{2} \leq \mu_{2}$ and fix $x_{1} \geq 1, x_{2} \geq 1$. If

$$
x_{1} r_{1}+x_{2} r_{2} \leq \frac{r_{1} \mu_{2}-r_{2} \mu_{1}}{\mu_{2}-\mu_{1}}
$$

and if $V\left(x_{1}, x_{2}-1 ; P_{1}\right) \geq V\left(x_{1}, x_{2}-1 ; P_{2}\right)$, and, for $x_{1} \neq 1$, if $V\left(x_{1}-1, x_{2} ; P_{1}\right) \geq V\left(x_{1}-\right.$ $\left.1, x_{2} ; P_{2}\right)$, then $V\left(x_{1}, x_{2} ; P_{1}\right) \geq V\left(x_{1}, x_{2} ; P_{2}\right)$.

\section{Proof:}

(i) We have

$$
\begin{aligned}
V\left(x_{1}, x_{2} ; P_{2}\right) & =\frac{\mu_{2} V\left(x_{1}, x_{2}-1, R\right)+x_{1} r_{1} V\left(x_{1}-1, x_{2} ; P_{2}\right)+\left(x_{2}-1\right) r_{2} V\left(x_{1}, x_{2}-1 ; P_{2}\right)}{\mu_{2}+x_{1} r_{1}+\left(x_{2}-1\right) r_{2}} \\
& \geq \frac{\mu_{2}\left(1+V\left(x_{1}, x_{2}-1, P_{1}\right)\right)+x_{1} r_{1} V\left(x_{1}-1, x_{2} ; P_{2}\right)+\left(x_{2}-1\right) r_{2} V\left(x_{1}, x_{2}-1 ; P_{1}\right)}{\mu_{2}+x_{1} r_{1}+\left(x_{2}-1\right) r_{2}}
\end{aligned}
$$

where the inequality follows because, for the first term, $V\left(x_{1}, x_{2}-1, R\right) \geq 1+V\left(x_{1}, x_{2}-1, P_{1}\right)$, and for the last term, either $x_{2}=1$, so the inequality is trivial, or $V\left(x_{1}, x_{2}-1 ; P_{2}\right) \geq$ $V\left(x_{1}, x_{2}-1 ; P_{1}\right)$. Similarly,

$$
\begin{aligned}
V\left(x_{1}, x_{2} ; P_{1}\right) & =\frac{\mu_{1} V\left(x_{1}-1, x_{2}, R\right)+\left(x_{1}-1\right) r_{1} V\left(x_{1}-1, x_{2} ; P_{1}\right)+r_{2} x_{2} V\left(x_{1}, x_{2}-1 ; P_{1}\right)}{\mu_{1}+\left(x_{1}-1\right) r_{1}+x_{2} r_{2}} \\
& \leq \frac{\mu_{1}\left(1+V\left(x_{1}-1, x_{2}, P_{2}\right)\right)+\left(x_{1}-1\right) r_{1} V\left(x_{1}-1, x_{2} ; P_{2}\right)+r_{2} x_{2} V\left(x_{1}, x_{2}-1 ; P_{1}\right)}{\mu_{1}+\left(x_{1}-1\right) r_{1}+x_{2} r_{2}} .
\end{aligned}
$$

Hence,

$$
\begin{aligned}
& V\left(x_{1}, x_{2} ; P_{2}\right)-V\left(x_{1}, x_{2} ; P_{1}\right) \\
& \geq\left(\frac{x_{1} r_{1}}{\mu_{2}+x_{1} r_{1}+\left(x_{2}-1\right) r_{2}}-\frac{\mu_{1}+\left(x_{1}-1\right) r_{1}}{\mu_{1}+\left(x_{1}-1\right) r_{1}+x_{2} r_{2}}\right) V\left(x_{1}-1, x_{2} ; P_{2}\right) \\
& \quad+\left(\frac{\mu_{2}+\left(x_{2}-1\right) r_{2}}{\mu_{2}+x_{1} r_{1}+\left(x_{2}-1\right) r_{2}}-\frac{x_{2} r_{2}}{\mu_{1}+\left(x_{1}-1\right) r_{1}+x_{2} r_{2}}\right) V\left(x_{1}, x_{2}-1 ; P_{1}\right) \\
&= \frac{\left(x_{1}-1\right) r_{1}\left(r_{2}-\mu_{2}\right)+\left(x_{2}-1\right) r_{2}\left(r_{1}-\mu_{1}\right)+r_{1} r_{2}-\mu_{1} \mu_{2}}{\left(\mu_{1}+\left(x_{1}-1\right) r_{1}+x_{2} r_{2}\right)\left(\mu_{2}+x_{1} r_{1}+\left(x_{2}-1\right) r_{2}\right)} \\
& \times\left(V\left(x_{1}-1, x_{2} ; P_{2}\right)-V\left(x_{1}, x_{2}-1 ; P_{1}\right)\right) \\
& \geq 0,
\end{aligned}
$$


where the first inequality follows from Condition (1) and the second follows from Lemma 1 and the conditions that $\mu_{1}<\mu_{2} \leq r_{2}$ and $\mu_{1} \leq r_{1}$.

(ii) We have

$$
\begin{aligned}
V\left(x_{1}, x_{2} ; P_{1}\right) & =\frac{\mu_{1} V\left(x_{1}-1, x_{2} ; R\right)+\left(x_{1}-1\right) r_{1} V\left(x_{1}-1, x_{2} ; P_{1}\right)+x_{2} r_{2} V\left(x_{1}, x_{2}-1 ; P_{1}\right)}{\mu_{1}+\left(x_{1}-1\right) r_{1}+x_{2} r_{2}} \\
& \geq \frac{\mu_{1}\left(1+V\left(x_{1}-1, x_{2} ; P_{2}\right)\right)+\left(x_{1}-1\right) r_{1} V\left(x_{1}-1, x_{2} ; P_{2}\right)+x_{2} r_{2} V\left(x_{1}, x_{2}-1 ; P_{1}\right)}{\mu_{1}+\left(x_{1}-1\right) r_{1}+x_{2} r_{2}}
\end{aligned}
$$

where the inequality follows because, for the first term, $V\left(x_{1}-1, x_{2} ; R\right) \geq 1+V\left(x_{1}-1, x_{2} ; P_{2}\right)$, and for the second term, either $x_{1}=1$, so the inequality is trivial, or $V\left(x_{1}-1, x_{2} ; P_{1}\right) \geq$ $V\left(x_{1}-1, x_{2} ; P_{2}\right)$. Similarly,

$$
\begin{aligned}
V\left(x_{1}, x_{2} ; P_{2}\right) & =\frac{\mu_{2} V\left(x_{1}, x_{2}-1 ; R\right)+x_{1} r_{1} V\left(x_{1}-1, x_{2} ; P_{2}\right)+\left(x_{2}-1\right) r_{2} V\left(x_{1}, x_{2}-1 ; P_{2}\right)}{\mu_{2}+x_{1} r_{1}+\left(x_{2}-1\right) r_{2}} \\
& \leq \frac{\mu_{2}\left(1+V\left(x_{1}, x_{2}-1 ; P_{1}\right)\right)+x_{1} r_{1} V\left(x_{1}-1, x_{2} ; P_{2}\right)+\left(x_{2}-1\right) r_{2} V\left(x_{1}, x_{2}-1 ; P_{1}\right)}{\mu_{2}+x_{1} r_{1}+\left(x_{2}-1\right) r_{2}} .
\end{aligned}
$$

Hence,

$$
\begin{aligned}
V\left(x_{1},\right. & \left.x_{2} ; P_{1}\right)-V\left(x_{1}, x_{2} ; P_{2}\right) \\
\geq & \left(\frac{\mu_{1}+\left(x_{1}-1\right) r_{1}}{\mu_{1}+\left(x_{1}-1\right) r_{1}+x_{2} r_{2}}-\frac{x_{1} r_{1}}{\mu_{2}+x_{1} r_{1}+\left(x_{2}-1\right) r_{2}}\right) V\left(x_{1}-1, x_{2} ; P_{2}\right) \\
& +\left(\frac{x_{2} r_{2}}{\mu_{1}+\left(x_{1}-1\right) r_{1}+x_{2} r_{2}}-\frac{\mu_{2}+\left(x_{2}-1\right) r_{2}}{\mu_{2}+x_{1} r_{1}+\left(x_{2}-1\right) r_{2}}\right) V\left(x_{1}, x_{2}-1 ; P_{1}\right) \\
= & \frac{\left(x_{1}-1\right) r_{1}\left(\mu_{2}-r_{2}\right)+\left(x_{2}-1\right) r_{2}\left(\mu_{1}-r_{1}\right)+\mu_{1} \mu_{2}-r_{1} r_{2}}{\left(\mu_{1}+\left(x_{1}-1\right) r_{1}+x_{2} r_{2}\right)\left(\mu_{2}+x_{1} r_{1}+\left(x_{2}-1\right) r_{2}\right)} \\
& \times\left(V\left(x_{1}-1, x_{2} ; P_{2}\right)-V\left(x_{1}, x_{2}-1 ; P_{1}\right)\right) \\
\geq & 0,
\end{aligned}
$$

where the first inequality follows from Condition (2) and the second follows from Lemma 1, and the conditions that $r_{1} \leq \mu_{1}<\mu_{2}$ and $r_{2} \leq \mu_{2}$.

Proposition 4 If $\mu_{1}<\mu_{2} \leq r_{2}$ and $\mu_{1} \leq r_{1}$, then for every $x_{1} \geq 0$, the optimal policy has a threshold, $t\left(x_{1}\right)$ (which may be infinite), such that for all $x_{2} \geq t\left(x_{1}\right)$, it is optimal to serve a type 2 job.

Proof: We prove the result by induction. Since serving a type 2 job is optimal for $x_{1}=0$, part (i) of Proposition 3 implies that if there is a state $(1, b)$ such that serving a type 2 job is optimal, then it is also optimal in all states $\left(1, x_{2}\right)$ such that $x_{2} \geq \max \left(b,\left(r_{1} \mu_{2}-\right.\right.$ $\left.\left.r_{2} \mu_{1}\right) /\left[r_{2}\left(\mu_{2}-\mu_{1}\right)\right]-r_{1} / r_{2}\right)$. Hence, there exists a threshold $t(1)$ such that for all $x_{2} \geq t(1)$, 
it is optimal serve a type 2 job in states $\left(1, x_{2}\right)$. Now suppose that serving a type 2 job is optimal in states $\left(a, x_{2}\right)$ for all $x_{2} \geq t(a)$. Then, if there is exists a state $(a+1, b)$ such that $b \geq t(a)-1$, where serving a type 2 job is optimal, then by part (i) of Proposition 3 , we see that serving a type 2 job is also optimal in all states $\left(a+1, x_{2}\right)$ such that $x_{2} \geq$ $\max \left(b,\left(r_{1} \mu_{2}-r_{2} \mu_{1}\right) /\left[r_{2}\left(\mu_{2}-\mu_{1}\right)\right]-r_{1}(a+1) / r_{2}\right)$. This completes the proof.

In Proposition 5, we show that if in addition to the conditions of Proposition 4 we also have that prioritizing type 2 jobs is agreeable with the $r \mu$ rule, then serving type 2 jobs is always optimal, i.e., the threshold in Proposition $4, t\left(x_{1}\right)$, is 1 for all $x_{1} \geq 0$.

Proposition 5 If $\mu_{1}<\mu_{2} \leq r_{2}, \mu_{1} \leq r_{1}$, and $r_{1} \mu_{1} \leq r_{2} \mu_{2}$, then $V\left(x_{1}, x_{2} ; P_{2}\right) \geq V\left(x_{1}, x_{2} ; P_{1}\right)$ for all $x_{i}=1,2, \ldots, m_{i}, i=1,2$.

Proof: First, note that Condition (1) is satisfied for all $x_{1}, x_{2} \geq 1$ since $\mu_{1}<\mu_{2}$ and $r_{1} \mu_{1} \leq r_{2} \mu_{2}$. Second, $r_{1} \mu_{1} \leq r_{2} \mu_{2}$ implies that $V\left(1,1 ; P_{1}\right) \leq V\left(1,1 ; P_{2}\right)$, and hence part (i) of Proposition 3, which holds since $\mu_{1}<\mu_{2} \leq r_{2}$ and $\mu_{1} \leq r_{1}$, yields that $V\left(x_{1}, 1 ; P_{1}\right) \leq$ $V\left(x_{1}, 1 ; P_{2}\right)$ for all $x_{1} \geq 1$. Finally, note that by convention, $V\left(0, x_{2} ; P_{1}\right)=0 \leq V\left(0, x_{2} ; P_{2}\right)$ for all $x_{2} \geq 1$. Combining this result with the fact that $V\left(x_{1}, 1 ; P_{1}\right) \leq V\left(x_{1}, 1 ; P_{2}\right)$ for all $x_{1} \geq 1$ complete the proof once we apply part (i) of Proposition 3 .

Proposition 6 If $r_{1} \leq \mu_{1}<\mu_{2}$ and $r_{2} \leq \mu_{2}$, then for every $x_{1} \geq 1$, the optimal policy has a threshold $t\left(x_{1}\right)$ such that for all $x_{2} \leq t\left(x_{1}\right)$, it is optimal to serve a type 1 job, and $t\left(x_{1}\right)$ is given by

$$
t\left(x_{1}\right)=\frac{r_{1} \mu_{2}-r_{2} \mu_{1}}{r_{2}\left(\mu_{2}-\mu_{1}\right)}-\frac{x_{1} r_{1}}{r_{2}} .
$$

Proof: First note that Condition (2) is equivalent to $x_{2} \leq t\left(x_{1}\right)$. For the condition to be satisfied at all (for some $x_{1} \geq 1, x_{2} \geq 1$ ), we must have $r_{2} \mu_{2} \leq r_{1} \mu_{1}$, which implies that $V\left(1,1 ; P_{1}\right) \geq V\left(1,1 ; P_{2}\right)$. Hence part (ii) of Proposition 3 yields that $V\left(1, x_{2} ; P_{1}\right) \geq$ $V\left(1, x_{2} ; P_{2}\right)$ for all $1 \leq x_{2} \leq t(1)$. Note also that by convention, $V\left(x_{1}, 0 ; P_{2}\right)=0 \leq$ $V\left(x_{1}, 0 ; P_{1}\right)$ for all $x_{1} \geq 1$. It is easy to see that $t\left(x_{1}\right)$ is non-increasing in $x_{1}$. Hence, for all $x_{2} \leq t\left(x_{1}\right)$, we have $V\left(x_{1}-1, x_{2} ; P_{1}\right) \geq V\left(x_{1}-1, x_{2} ; P_{2}\right)$. Then, applying part (ii) of Proposition 3 completes the proof.

Suppose now that $\mu_{1}<\mu_{2}$ and $r_{1}>r_{2}$, i.e., type 1 jobs, which are more time-critical, have larger mean service times. For this case, Propositions 5 and 6 yield the following conclusions: 
- By Proposition 5, if $r_{i} \geq \mu_{i}$ for $i=1,2$, and $r_{2} \mu_{2} \geq r_{1} \mu_{1}$, then it is always optimal to serve a type 2 job, which is less time-critical and has a smaller mean service time than a type 1 job. The first condition means that both types of jobs abandon the system at higher rates than their service rates. On the other hand, the second condition is the necessary and sufficient condition for the optimality of serving a type 2 job when $x_{1}=x_{2}=1$. Hence, if jobs abandon the system at higher rates (relative to service rates) and if it is better to serve a type 2 job when there is only one of each type, then type 2 jobs should always be given priority regardless of the system state.

- Proposition 6 implies that if $r_{i} \leq \mu_{i}$ for $i=1,2$, then it is optimal to serve a type 1 job, which is more time-critical and has a larger mean service time, when the number of jobs in the system satisfy Condition (2). Note that Condition (2) generally holds when $x_{1}$ and $x_{2}$ are small. Hence, Proposition 6 implies that when service is fast (relative to abandonments) and the number of jobs in the system are sufficiently small, then the time-critical but slower jobs should be given priority for service.

We end this section with a conjecture. Based on extensive numerical experiments, we believe that Proposition 5 holds under a set of less restrictive conditions:

Conjecture 1 If $\mu_{1}<\mu_{2}$ and $r_{1} \mu_{1} \leq r_{2} \mu_{2}$, then $V\left(x_{1}, x_{2} ; P_{2}\right) \geq V\left(x_{1}, x_{2} ; P_{1}\right)$ for all $x_{i}=$ $1,2, \ldots, m_{i}, i=1,2$.

In other words, we conjecture that as long as the $r \mu$ rule is agreeable with SEPT, the $r \mu$ rule is optimal among all policies in $\Pi$.

Although we were able to prove this conjecture for states $\left(1, x_{2}\right)$, where $x_{2}=1,2, \ldots, m_{2}$, and states $\left(x_{1}, 1\right)$, where $x_{1}=1,2, \ldots, m_{1}$, proving it in its most general form appears to be a significant challenge. We defer the proof of Proposition 7 to the Appendix.

Proposition 7 The r $\mu$ rule is optimal among all policies within $\Pi$ when it is agreeable with SEPT and either $x_{1}$ or $x_{2}$ is at most 1.

\section{Heuristic policies}

In this section, we propose two new heuristic policies, namely the triangular and rectangular heuristics, for state-dependent job prioritization decisions when there are two types of jobs in the system. We also describe two other heuristics both of which are static policies in the sense that under these policies priorities do not change with the system state. In Section 6 , we compare the performances of these four heuristics along with the performance of the optimal policy by means of a numerical study. 
When time-critical jobs have shorter service times (i.e., $r_{1} \geq r_{2}$ and $\mu_{1} \geq \mu_{2}$ using the notation of Section 4), Theorem 1 shows that the optimal policy should always give priority to time-critical jobs (type 1 jobs) regardless of the system state. On the other hand, when time-critical jobs have longer service times (i.e., $r_{1}>r_{2}$ and $\mu_{1}<\mu_{2}$ ), it is generally not clear what the best prioritization policy is (except for the cases identified in Propositions 5 and 6 ). Therefore, we develop heuristic policies for this particular case, and assume that $r_{1}>r_{2}$ and $\mu_{1}<\mu_{2}$ in the following discussion. Below, we describe how the heuristic policies work when service times and lifetimes are exponentially distributed. However, these heuristics can be also applied in more general settings as we discuss in Section 6.1.2.

The heuristic procedures that we propose, namely the triangular and rectangular heuristics, are primarily based on the structural results given in Section 4 and our observations on the structure of the optimal policy from numerical experiments. We observed from our experiments that the optimal policy divides the state space into at most two regions as shown in Figure 1. In general, the optimal policy does not have any obvious monotonic structure as Figure 1 reveals. However, in many cases, the optimal policy is monotone in the number of type 1 and type 2 jobs. More specifically, if it is optimal to serve a type 1 job in state $\left(x_{1}, x_{2}\right)$, it is also optimal to serve a type 1 job in state $\left(x_{1}-1, x_{2}\right)$ or in state $\left(x_{1}, x_{2}-1\right)$; similarly, if it is optimal to serve a type 2 job in state $\left(x_{1}, x_{2}\right)$, it is also optimal to serve a type 2 job in state $\left(x_{1}, x_{2}+1\right)$ or in state $\left(x_{1}+1, x_{2}\right)$. The triangular heuristic mimics this behavior of the optimal policy with some support from our theoretical results. The rectangular heuristic is based on the triangular heuristic, but is easier to implement.

We next describe these heuristic policies in detail.

(i) Triangular heuristic: This heuristic is primarily based on Proposition 3. Condition (1), or equivalently Condition (2), of Proposition 3 divides the state space into two regions, one of which roughly has the shape of a triangle. When the system is in state $\left(x_{1}, x_{2}\right)$, the triangular heuristic gives priority to type 1 jobs if $\left(x_{1}, x_{2}\right)$ falls inside the triangle (i.e., if $\left(x_{1}, x_{2}\right)$ satisfies Condition (2)), and it gives priority to type 2 jobs otherwise. Note that Proposition 3 does not give a complete characterization of the optimal policy, but whether Condition (1) or (2) holds in Proposition 3 can be seen as an indicator of preference towards one type over the other. Figure 2 (a) shows how the triangular heuristic works for the example studied in Figure 1.

The triangular heuristic is also insightful. To illustrate, we first rewrite Condition (2) as follows:

$$
\frac{\left(x_{1}-1\right) r_{1}+x_{2} r_{2}}{\mu_{1}} \leq \frac{x_{1} r_{1}+\left(x_{2}-1\right) r_{2}}{\mu_{2}} .
$$

The left-hand side of Condition (3) is the mean number of abandonments during the 
service of a type 1 job, whereas the right-hand side is the mean number of abandonments during the service of a type 2 job in the same state. Hence, in some sense, the triangular heuristic (myopically) gives priority to jobs with a smaller mean number of abandonments during service.

Note also that the triangular heuristic is in agreement with all of our analytical results on the characterization of the optimal policy. More specifically, when $r_{1} \mu_{1} \leq r_{2} \mu_{2}$ and $\mu_{1}<\mu_{2}$, the heuristic gives priority to type 2 jobs independently of the system state, which is consistent with Propositions 5 and 7, and Conjecture 1. Similarly, the heuristic is also in agreement with Proposition 6. Finally, when $r_{1} \geq r_{2}$ and $\mu_{1} \geq \mu_{2}$, the heuristic gives higher priority to type 1 jobs in all states, which is consistent with Theorem 1.

Figure 2: Examples on the structure of the triangular and rectangular heuristics.

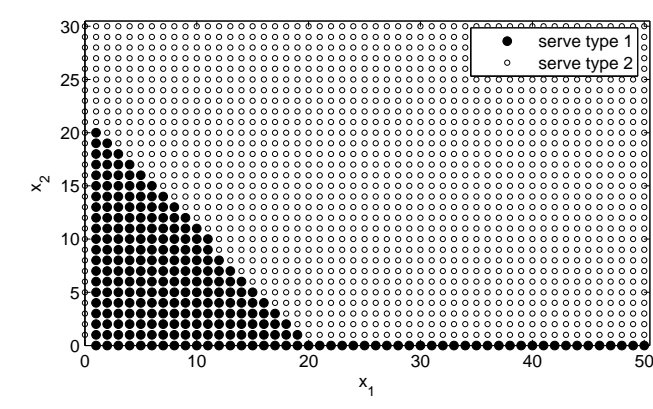

(a) Triangular heuristic

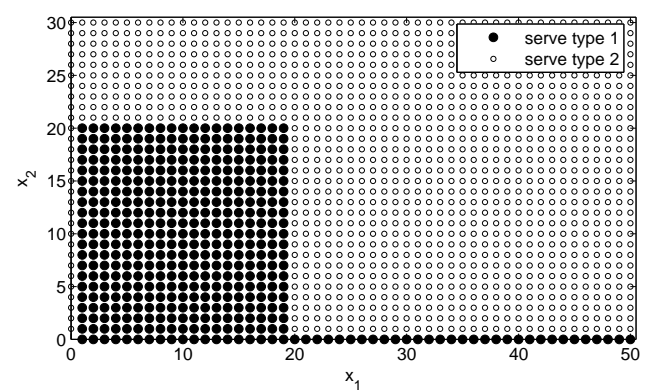

(b) Rectangular heuristic

(ii) Rectangular heuristic: The rectangular heuristic is based on the triangular heuristic and works similarly. The idea is simply to expand the triangular region associated with the triangular heuristic to a rectangle by adding another point to the existing three points and connecting the four points on the state space. Figure 2 (b) shows how the rectangular heuristic works for the case studied in Figure 1. One advantage of the rectangular heuristic is its simple structure. It is completely characterized by two threshold values, i.e., the length and the width of the rectangle. More precisely, in a given state $\left(x_{1}, x_{2}\right)$, if $r_{1}>r_{2}$ and $\mu_{1}<\mu_{2}$, then the rectangular heuristic gives priority to type 1 jobs if and only if $1 \leq x_{1} \leq \min \left\{m_{1}, T_{1}\right\}$ and $1 \leq x_{2} \leq \min \left\{m_{2}, T_{2}\right\}$, where

$$
T_{1}=\frac{\mu_{2}\left(r_{1}-r_{2}\right)}{r_{1}\left(\mu_{2}-\mu_{1}\right)}
$$

and

$$
T_{2}=\frac{\mu_{1}\left(r_{1}-r_{2}\right)}{r_{2}\left(\mu_{2}-\mu_{1}\right)} .
$$


$T_{1}$ is obtained by plugging $x_{1}=T_{1}$ and $x_{2}=1$ in Condition (2) and solving it as an equality, and $T_{2}$ is obtained similarly by plugging $x_{1}=1$ and $x_{2}=T_{2}$ in the same inequality. Since we assume that $r_{1}>r_{2}$ and $\mu_{1}<\mu_{2}, T_{1}$ and $T_{2}$ will always be nonnegative. Note that when $r_{1} \mu_{1} \leq r_{2} \mu_{2}$ and $\mu_{1}<\mu_{2}$, the rectangular heuristic prioritizes type 2 jobs in all states for which $x_{2} \geq 1$, which is consistent with Propositions 5 and 7 , and Conjecture 1. On the other hand, if $r_{1} \mu_{1}>r_{2} \mu_{2}$ and $\mu_{1}<\mu_{2}$, the rectangular heuristic gives a state-dependent policy. In this case, the threshold for type 2 jobs is higher than the threshold for type 1 jobs, i.e., $1<T_{1}<T_{2}$.

(iii) $r \mu$-heuristic: This heuristic is studied by Glazebrook, Ansell, Dunn, and Lumley [14] for a slightly different version of our problem. More specifically, the authors consider scheduling of jobs in a clearing system with $N \geq 2$ jobs initially. Each job is characterized by its service rate $\mu$, abandonment rate $r$, and the positive reward $\theta$ that it brings after service completion. (See Section 2 for more details on the paper by Glazebrook et al. [14].) The authors propose a heuristic that schedules the jobs in a non-increasing order of the index $\theta r \mu$. For our problem, their heuristic is equivalent to the $r \mu$ rule.

Glazebrook et al. [14] prove that the $r \mu$-heuristic, which is a state-independent policy, is asymptotically optimal as the abandonment rates approach zero when the times to abandonment are exponentially distributed. Note that the $r \mu$-heuristic is identical to the triangular and rectangular heuristics, and is consistent with Conjecture 1, when it is also agreeable with SEPT. Also, when $r_{1} \geq r_{2}$ and $\mu_{1} \geq \mu_{2}$, it gives higher priority to type 1 jobs, which is consistent with Theorem 1.

(iv) Time-Critical First (TCF) heuristic: This heuristic simply gives priority to jobs with higher abandonment rates ignoring the service rates as well as the system state. To be more precise, it gives priority to type 1 jobs if and only if $r_{1}>r_{2}$. This heuristic is not expected to perform well in most cases. However, it is still included as a benchmark policy since it is commonly applied in daily operations, especially in situations where the pressure of making quick decisions with lack of prior planning leads to giving priorities to more urgent jobs with possibly long processing times.

\section{$6 \quad$ Numerical results}

In this section, we provide numerical results on the performance of the heuristic policies described in Section 5 for the case where time-critical jobs have longer service times. In Section 6.1, we compare the performance of the heuristic policies under various randomly 
generated scenarios and in Section 6.2, we take a closer look at the effects of certain input parameters on the performance of the heuristics.

\subsection{Comparison of heuristic policies}

We performed two sets of numerical experiments. In the first set, we considered jobs with exponential service times and lifetimes, whereas in the second set, we considered jobs with deterministic service times and lifetimes that have Weibull distribution. For both sets of experiments, we can obtain the optimal policy by solving the backward dynamic programming recursions, and hence we can compare the performance of the heuristic policies with the optimal performance.

\subsubsection{Exponential service times and lifetimes}

We consider systems where the service times and lifetimes for type $i$ jobs are exponentially distributed with respective rates $\mu_{i}>0$ and $r_{i}>0$ for $i=1,2$. Since we would like to study many different scenarios with a wide range of system parameters, we have sampled the system parameters randomly. More specifically, the initial number of jobs $m_{i}$ for each type $i \in\{1,2\}$ is drawn independently from a discrete uniform distribution over the set $\{1,2, \ldots, 100\}$. We have also generated the service rate $\mu_{i}$ of each job type $i$ from a (continuous) uniform distribution with range $[0.5,2.0]$. Similarly, we have generated the abandonment rate $r_{i}$ of each job type $i$ from a uniform distribution for $i=1,2$. We have considered five subsets of experiments depending on the range of the abandonment rates, namely [2.0,5.0], [0.5, 2.0], $[0.1,0.5],[0.01,0.1]$, and $[0.005,0.001]$. (Note that in the first two subsets, jobs are very critical since the abandonment rates are either larger than or close to the service rates. On the other hand, in the last three subsets, jobs are not very critical since the abandonment rates are smaller than service rates.) For each subset, we generated 5,000 random scenarios where $r_{1}>r_{2}$ and $\mu_{1}<\mu_{2}$. We excluded the cases for which we already know what the optimal policy is, based on Proposition 5. For each scenario, we calculated the expected number of jobs taken into service under each of the four heuristic policies and the optimal policy. Then, we computed the percentage deviation of the performance of each heuristic from that of the optimal policy. Based on these 5,000 percentage deviations, we constructed a $95 \%$ confidence interval (C.I.) on the mean; estimated the median, lower and upper quartiles; and determined the maximum percentage deviation, i.e., the worst performance. For each heuristic, we also calculated the number of times that the heuristic provided the best performance among the four heuristics. These results are presented in Table 1. (Note that the values in the last column of Table 1 do not add up to 5,000 for each subset of experiments due to ties among heuristics.) 


\begin{tabular}{|c|c|c|c|c|c|c|}
\hline Heuristic & $\begin{array}{c}95 \% \text { C.I. } \\
\text { on the mean }\end{array}$ & Lower quartile & Median & Upper quartile & Maximum & Best heuristic in \\
\hline & \multicolumn{6}{|c|}{$r_{i} \sim$ Uniform $[2.0,5.0]$} \\
\hline Triangular & $0.011 \pm 0.001$ & 0.000 & 0.001 & 0.009 & 2.064 & 4297 scenarios \\
\hline Rectangular & $0.013 \pm 0.001$ & 0.000 & 0.001 & 0.011 & 1.946 & 3275 scenarios \\
\hline$r \mu$ & $7.600 \pm 0.179$ & 2.340 & 5.950 & 11.512 & 33.7632 & 146 scenarios \\
\hline \multirow[t]{2}{*}{ TCF } & $7.600 \pm 0.179$ & 2.340 & 5.950 & 11.512 & 33.7632 & 146 scenarios \\
\hline & \multicolumn{6}{|c|}{$r_{i} \sim$ Uniform $[0.5,2.0]$} \\
\hline Triangular & $0.053 \pm 0.007$ & 0.000 & 0.000 & 0.011 & 6.304 & 3990 scenarios \\
\hline Rectangular & $0.042 \pm 0.006$ & 0.000 & 0.000 & 0.007 & 6.218 & 4628 scenarios \\
\hline$r \mu$ & $4.873 \pm 0.207$ & 0.000 & 0.000 & 7.928 & 37.255 & 2554 scenarios \\
\hline \multirow[t]{2}{*}{$\mathrm{TCF}$} & $18.214 \pm 0.386$ & 6.189 & 15.656 & 28.153 & 57.445 & 179 scenarios \\
\hline & \multicolumn{6}{|c|}{$r_{i} \sim$ Uniform $[0.1,0.5]$} \\
\hline Triangular & $0.425 \pm 0.031$ & 0.000 & 0.000 & 0.221 & 11.264 & 3678 scenarios \\
\hline Rectangular & $0.372 \pm 0.028$ & 0.000 & 0.000 & 0.200 & 11.134 & 4325 scenarios \\
\hline$r \mu$ & $3.072 \pm 0.151$ & 0.000 & 0.000 & 4.111 & 30.303 & 3084 scenarios \\
\hline \multirow[t]{2}{*}{ TCF } & $13.049 \pm 0.330$ & 2.668 & 10.055 & 20.529 & 52.741 & 737 scenarios \\
\hline & \multicolumn{6}{|c|}{$r_{i} \sim$ Uniform $[0.01,0.1]$} \\
\hline Triangular & $2.162 \pm 0.102$ & 0.000 & 0.047 & 2.978 & 21.659 & 2893 scenarios \\
\hline Rectangular & $2.077 \pm 0.099$ & 0.000 & 0.033 & 2.790 & 21.636 & 2923 scenarios \\
\hline$r \mu$ & $0.581 \pm 0.047$ & 0.000 & 0.000 & 0.001 & 20.678 & 4134 scenarios \\
\hline \multirow[t]{2}{*}{$\mathrm{TCF}$} & $4.117 \pm 0.167$ & 0.000 & 1.161 & 6.026 & 38.170 & 2160 scenarios \\
\hline & \multicolumn{6}{|c|}{$r_{i} \sim$ Uniform $[0.005,0.01]$} \\
\hline Triangular & $0.340 \pm 0.025$ & 0.000 & 0.000 & 0.016 & 7.939 & 3822 scenarios \\
\hline Rectangular & $0.335 \pm 0.025$ & 0.000 & 0.000 & 0.014 & 7.939 & 3831 scenarios \\
\hline$r \mu$ & $0.043 \pm 0.006$ & 0.000 & 0.000 & 0.000 & 3.573 & 4681 scenarios \\
\hline $\mathrm{TCF}$ & $3.189 \pm 0.106$ & 0.096 & 1.773 & 4.989 & 22.323 & 1199 scenarios \\
\hline
\end{tabular}

Table 1: Performance of the heuristics for exponential service times and lifetimes (in terms of the percentage deviation from the optimal performance) when $\mu_{i} \sim$ Uniform $[0.5,2.0]$ and $m_{i} \sim$ Uniform $\{1,2, \ldots, 100\}$, for $i=1,2$.

From Table 1, it can be seen that the performance of the heuristics depend on how fast the jobs abandon the system. When jobs are very critical (i.e., when $r_{i}$ 's are larger than or similar to $\mu_{i}$ 's), then the triangular and rectangular heuristics clearly provide the best performance. On the other hand, when jobs are not very critical (i.e., when $r_{i}$ 's are small compared to $\mu_{i}$ 's), then the $r \mu$-heuristic has the best performance. This is an expected result since $r \mu$-heuristic was shown to be asymptotically optimal as the abandonment rates approach to zero, see Glazebrook et al. [14]. However, Table 1 shows that all heuristics (except for the TCF heuristic, which gives the worst overall performance in all cases) perform reasonably well when abandonment rates are very small. This is not surprising because when abandonment rates are very small, jobs are likely to stay in the system for a long time, which makes the difference between the performance of any two non-idling policy less significant.

Table 1 also reveals that the worst performance for the triangular and rectangular heuristics is approximately $22 \%$, whereas the worst performance is $37 \%$ and $57 \%$ for the $r \mu$-heuristic and the TCF heuristic, respectively. This suggests that overall, the triangular and rectangular heuristics seem to be more robust since they do not perform very badly even over the parameter regions where the $r \mu$-heuristic gives a better performance. 


\subsubsection{Weibull lifetimes and deterministic service times}

In this section, our objective is to test the performance of the heuristics under a nonexponential setting. More specifically, we consider systems where the service time of a type $i$ job is deterministic and equal to $1 / \mu_{i}$, whereas its lifetime has a Weibull distribution with shape parameter $\alpha_{i}>0$ and scale parameter $\beta_{i}>0$. Then, the abandonment rates are given by $r_{i}=\alpha_{i} /\left(\beta_{i} \Gamma\left(1 / \alpha_{i}\right)\right)$ for $i=1,2$, where $\Gamma(i)$ is the gamma function. The Weibull distribution is commonly used in modeling lifetimes of humans, and possesses some nice properties such as the possibility of an increasing failure rate, see, e.g., Section 2.2 .2 in Hougaard [15]. (We choose service times to be deterministic since this allows us to compute the performance of the optimal policy.)

In Section 5, we described the heuristics under the assumption that the service times and lifetimes are exponentially distributed. Generalization of these heuristics to systems with non-exponential lifetimes is not immediate due to the lack of the memoryless property. Thus, we propose and test the following generalization to all four heuristics: At each decision epoch, i.e., at the end of each service completion, we calculate the updated abandonment rate, which is the reciprocal of the mean remaining lifetime, for each job type. Let $r_{i}(t)$ denote the updated abandonment rate for job type $i \in\{1,2\}$ at time $t \geq 0$. (Note that $r_{i}(0)=r_{i}$.) Then, at each decision epoch, the heuristics use the same decision rules as before except that these $r_{i}(t)$ values are used instead of $r_{i}$ 's. (If the order of $r_{i}(t)$ 's switch at a decision epoch such that the type with the faster service becomes time-critical, then we apply the optimal policy characterized by Theorem 1.) For the Weibull distribution with shape parameter $\alpha_{i}$ and scale parameter $\beta_{i}, i \in\{1,2\}$, we obtain that

$$
r_{i}(t)=\frac{\alpha_{i}}{\beta_{i} \Gamma\left(1 / \alpha_{i},\left(t / \beta_{i}\right)^{\alpha_{i}}\right)} e^{-\left(t / \beta_{i}\right)^{\alpha_{i}}}
$$

where $\Gamma(a, b)$, which is the incomplete gamma function, is defined as

$$
\Gamma(a, b)=\int_{b}^{\infty} u^{a-1} e^{-u} d u
$$

for $a>0$ and $b \geq 0$.

In our experiments presented in this section, the initial number of jobs $m_{i}$ for each type $i$ is drawn independently from a discrete uniform distribution over the set $\{1,2, \ldots, 20\}$. We have generated the service rate $\mu_{i}$ of each job type $i$ from a (continuous) uniform distribution with range $[0.5,2.0]$. For each job type $i \in\{1,2\}$, we let $\alpha_{i}=1.5$ and then generate the initial abandonment rate $r_{i}(0)$ from a uniform distribution. We considered five subsets of experiments depending on the range of the initial abandonment rate, namely $[2.0,5.0]$, $[0.5,2.0],[0.1,0.5],[0.01,0.1]$, and $[0.005,0.01]$. For each subset, we generated 5,000 random 
scenarios where $r_{1}(0)>r_{2}(0)$ and $\mu_{1}<\mu_{2}$. (Since in this example the lifetime distributions for both types of jobs are Weibull with the same shape parameter $\alpha$, having $r_{1}(0)>r_{2}(0)$ implies that the lifetime of a type 1 job is smaller than the lifetime of a type 2 job in the sense of hazard rate ordering, i.e., $r_{1}(t) \geq r_{2}(t)$ for all $t \geq 0$.) We computed the performance of each heuristic as in the exponential case, see Section 6.1.1. The results are summarized in Table 2.

\begin{tabular}{|c|c|c|c|c|c|c|}
\hline Heuristic & $\begin{array}{c}95 \% \text { C.I. } \\
\text { on the mean }\end{array}$ & Lower quartile & Median & Upper quartile & Maximum & Best heuristic in \\
\hline & \multicolumn{6}{|c|}{ 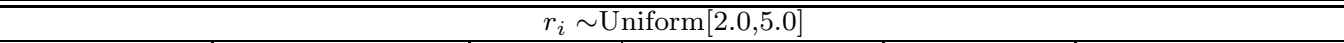 } \\
\hline Triangular & $0.015 \pm 0.004$ & 0.000 & 0.000 & 0.000 & 3.490 & 4943 scenarios \\
\hline Rectangular & $0.027 \pm 0.007$ & 0.000 & 0.000 & 0.000 & 8.688 & 4796 scenarios \\
\hline$r \mu$ & $3.089 \pm 0.203$ & 0.000 & 0.000 & 1.258 & 46.922 & 3032 scenarios \\
\hline \multirow[t]{2}{*}{ TCF } & $19.609 \pm 0.488$ & 2.755 & 15.289 & 34.561 & 63.145 & 207 scenarios \\
\hline & \multicolumn{6}{|c|}{$\overline{r_{i} \sim \text { Uniform }[0.5,2.0]}$} \\
\hline Triangular & $0.163 \pm 0.019$ & 0.000 & 0.000 & 0.051 & 14.010 & 4609 scenarios \\
\hline Rectangular & $0.175 \pm 0.019$ & 0.000 & 0.000 & 0.056 & 14.010 & 4024 scenarios \\
\hline$r \mu$ & $4.534 \pm 0.191$ & 0.000 & 0.002 & 7.609 & 34.212 & 2505 scenarios \\
\hline \multirow[t]{2}{*}{$\mathrm{TCF}$} & $17.004 \pm 0.384$ & 5.033 & 14.440 & 26.546 & 62.378 & 428 scenarios \\
\hline & \multicolumn{6}{|c|}{ 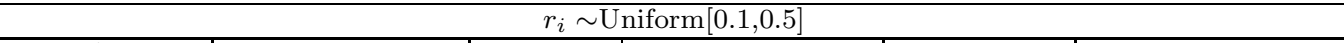 } \\
\hline Triangular & $1.129 \pm 0.066$ & 0.000 & 0.007 & 1.057 & 19.745 & 3320 scenarios \\
\hline Rectangular & $1.006 \pm 0.063$ & 0.000 & 0.003 & 0.813 & 19.745 & 3639 scenarios \\
\hline$r \mu$ & $1.242 \pm 0.077$ & 0.000 & 0.000 & 0.738 & 21.399 & 3626 scenarios \\
\hline \multirow[t]{2}{*}{$\mathrm{TCF}$} & $7.502 \pm 0.261$ & 0.000 & 3.613 & 11.815 & 49.918 & 1643 scenarios \\
\hline & \multicolumn{6}{|c|}{$r_{i} \sim$ Uniform $[0.01,0.1]$} \\
\hline Triangular & $2.398 \pm 0.112$ & 0.000 & 0.405 & 3.030 & 25.039 & 1997 scenarios \\
\hline Rectangular & $2.313 \pm 0.111$ & 0.000 & 0.326 & 2.918 & 25.039 & 2076 scenarios \\
\hline$r \mu$ & $0.017 \pm 0.003$ & 0.000 & 0.000 & 0.000 & 3.982 & 4662 scenarios \\
\hline \multirow[t]{2}{*}{$\mathrm{TCF}$} & $0.614 \pm 0.047$ & 0.000 & 0.000 & 0.309 & 23.985 & 3297 scenarios \\
\hline & \multicolumn{6}{|c|}{ 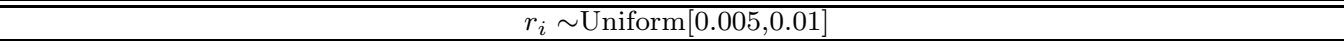 } \\
\hline Triangular & $0.208 \pm 0.012$ & 0.000 & 0.001 & 0.203 & 5.266 & 2502 scenarios \\
\hline Rectangular & $0.201 \pm 0.012$ & 0.000 & 0.000 & 0.188 & 5.266 & 2555 scenarios \\
\hline$r \mu$ & $0.008 \pm 0.001$ & 0.000 & 0.000 & 0.000 & 0.514 & 4446 scenarios \\
\hline TCF & $0.135 \pm 0.008$ & 0.000 & 0.000 & 0.131 & 3.259 & 2673 scenarios \\
\hline
\end{tabular}

Table 2: Performance of the heuristics for deterministic service times and Weibull lifetimes (in terms of the percentage deviation from the optimal performance) when $\alpha_{i}=1.5$, $\mu_{i} \sim$ Uniform $[0.5,2.0]$, and $m_{i} \sim$ Uniform $\{1,2, \ldots, 20\}$, for $i=1,2$.

Perhaps the most important observation from Table 2 is that the heuristics that we developed for the exponential case also perform well in a non-exponential setting. Furthermore, the general behavior of the heuristics does not appear to be much affected by the distributional assumption. More specifically, as in the case with exponential distributions, triangular and rectangular heuristics still provide the best performance when jobs abandon the system with high rates while the $r \mu$-heuristic is the best when abandonment rates are small. Interestingly, the TCF heuristic yields a more pronounced performance in extreme cases when compared with its performance under exponential distributions. To be more specific, when abandonment rates are large, TCF's performance is worse than its performance for the exponential case, whereas when abandonment rates are small, its performance is better than 
its performance for the exponential case.

\subsection{Effects of some system parameters on the performance of heuristics}

In this section, we investigate the effects of two system parameters on the performances of the four heuristic policies under exponential lifetime and service time distributions. These two parameters are the initial total number of jobs, $m_{1}+m_{2}$, and the ratio $\phi=r_{2} \mu_{2} /\left(r_{1} \mu_{1}\right)$, which can be considered as a measure of similarity between the two job types. To observe the effect of $m_{1}+m_{2}$, we first computed $m_{1}+m_{2}$ for each of the 5,000 random scenarios (generated for the experiments presented in Section 6.1.1) and sorted the scenarios in an ascending order of their $m_{1}+m_{2}$ values. Then, we computed the moving average (with a window size of 1,500) of the percentage deviation of each heuristic from the optimal policy. To observe the effect of $\phi$, we followed the same procedure except that the window size for the moving average was set to 500. The moving average plots for $m_{1}+m_{2}$ and $\phi$ are given in Figures 3 and 4, respectively. In the interest of space, we present the plots for only three subsets of our experiments, namely the ones where the ranges for the abandonment rates are $[0.5,2.0],[0.1,0.5]$, and $[0.01,0.1]$.

From Figure 3, it can be seen that the performances of the $r \mu$ and TCF heuristics worsen with the total number of jobs in the system in all three cases considered. On the other hand, the performances of the triangular and rectangular heuristics have a tendency to improve as the number of jobs in the system increases when jobs abandon the system at a relatively high rate. Together with our conclusions from Section 6.1, this suggests that the triangular and rectangular heuristics are well-suited for worst-case scenarios, where the system is overwhelmed with a large number of jobs that abandon the system quickly. Such scenarios can be typically realized in the wake of mass casualty incidents, which cause significant number of casualties who are in need of immediate care.

Figure 4 shows the relationship between the performance of the heuristics and the parameter $\phi$. First, note that the moving average plots for the triangular, rectangular, and $r \mu$ heuristics hit zero after a certain point (when $\phi$ is around one). This is due to the fact that these three heuristics are in fact equivalent and furthermore optimal (which is a numerical observation) when $r_{2} \mu_{2} \geq r_{1} \mu_{1}$ and $\mu_{1}<\mu_{2}$ under exponential distributions, see Conjecture 1.

As we have discussed in Section 6.1, the triangular and rectangular heuristics perform very well when jobs have high abandonment rates. Figure 4 supports this observation but also suggests that in some cases these heuristics should be preferred even when the abandonment rates are small. To see this, first note that regardless of whether abandonment rates are small 
Figure 3: Moving average plots of percentage deviations of heuristics from the optimal with respect to the number of scenarios that are ordered according to the increasing initial total number of jobs $m_{1}+m_{2}$.

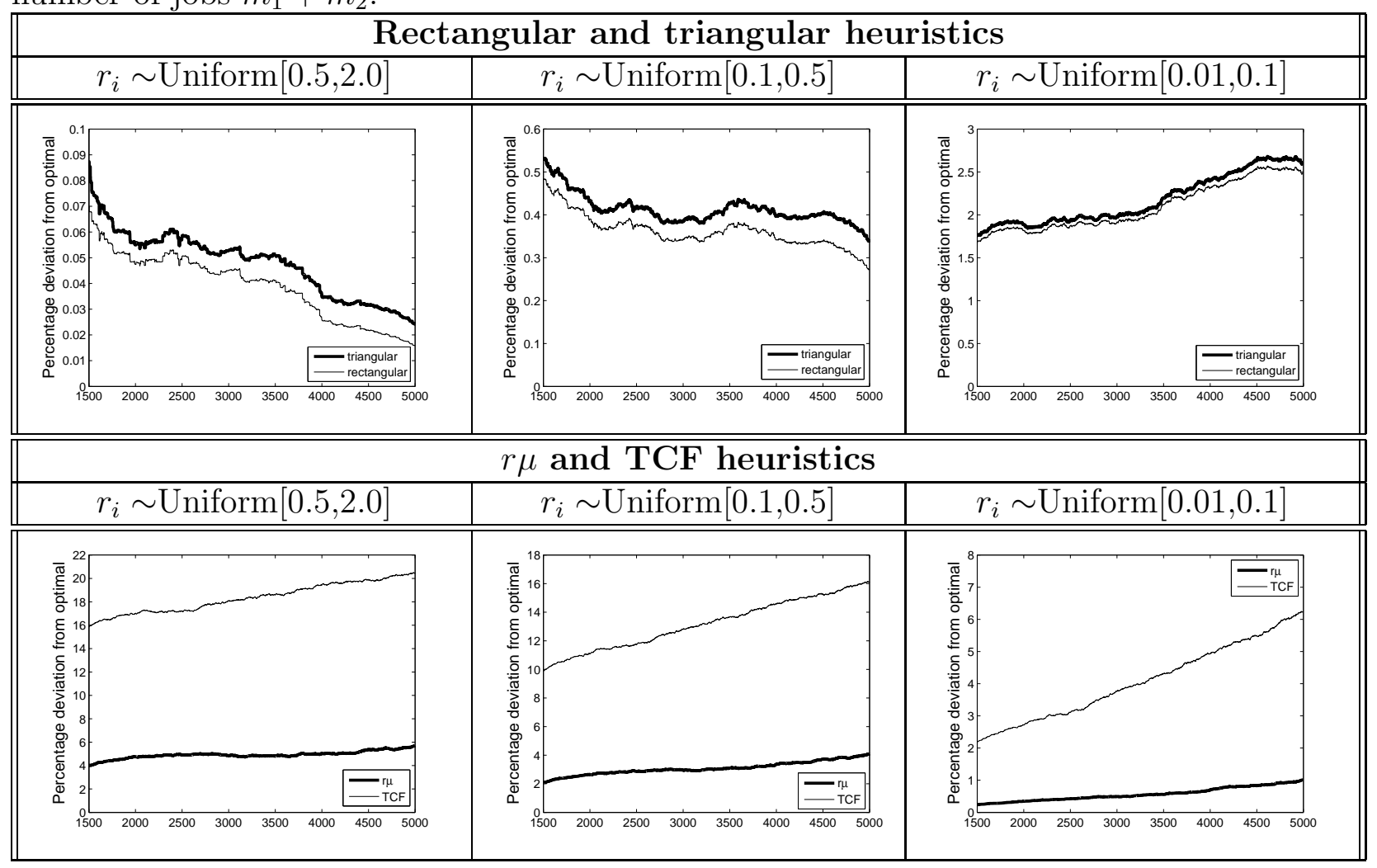

or large, the triangular and rectangular heuristics provide their worst performances when $r_{2} \mu_{2}$ is small; however, they perform increasingly well as $r_{2} \mu_{2}$ gets closer to $r_{1} \mu_{1}$. (Note that $r_{2} \mu_{2}$ is close to $r_{1} \mu_{1}$ around the middle of the $x$-axis.) On the other hand, the $r \mu$ heuristic provides its worst performance when $r_{2} \mu_{2} /\left(r_{1} \mu_{1}\right)$ approaches one. Now comparing the two plots for which $r_{i} \in[0.01,0.1]$ in Figure 4, we see that even when the abandonment rates are small but $r_{2} \mu_{2} /\left(r_{1} \mu_{1}\right)$ is close to one, the triangular and rectangular heuristics yield a better average performance than the $r \mu$ and TCF heuristics, and thus are preferable.

\section{Conclusions}

We considered a clearing system with a single server and a finite number of jobs that may abandon the system before receiving service. For such a system, we studied the optimal and near-optimal scheduling of jobs, which are characterized by their service time and lifetime distributions, with the objective of minimizing the total number of abandonments. We are mainly motivated by the patient triage problem, which arises in the aftermath of mass casualty events. Our question is: Given the operating/treatment time and lifetime distributions 
Figure 4: Moving average plots of percentage deviations of heuristics from the optimal with respect to the number of scenarios that are ordered according to the increasing value of $r_{2} \mu_{2} /\left(r_{1} \mu_{1}\right)$.

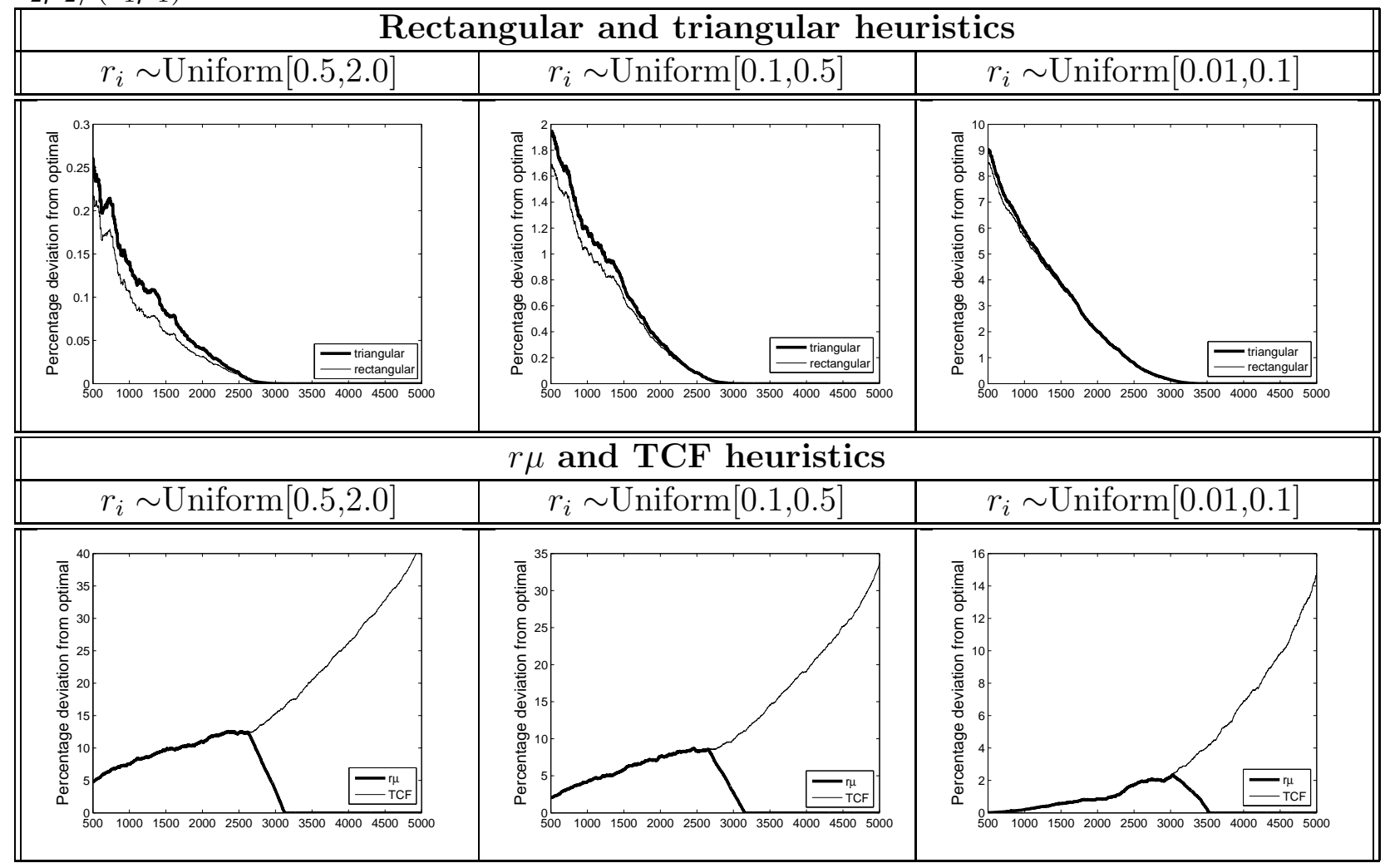

of different patients with different injuries and also given the number of patients, how should the patients be admitted to a scarce resource (e.g., an operating room) so as to maximize the total number of patients saved? In practice, there is not a simple answer to this question since each mass casualty event is unique with challenges that typically cannot be anticipated, and such events require prompt decisions from human beings working in chaotic environments. Thus, in this work, we provide some general insights into the problem by identifying the characteristics of effective prioritization decision rules under different operating conditions.

Using sample path arguments for general service time and lifetime distributions, and a stochastic dynamic programming approach for exponential service time and lifetime distributions, we identified characteristics of the optimal policy analytically for several cases. For example, we showed that if jobs can be ordered in such a way that the job with the shortest lifetime (in the sense of hazard rate orders) also has the shortest service time (in the sense of likelihood ratio orders), then it should always be given priority for service, regardless of the state of the system. This result makes sense intuitively, but more importantly, it provides us with a criterion as to what makes a job a top-priority job. For the patient triage problem, this implies that regardless of how many patients are in need of treatment, a patient with a 
certain injury can be given the highest priority if we know that, without any medical intervention, his/her lifetime will be shorter than any other patient in the sense of hazard rate ordering, and the operation for that specific injury takes a shorter time than all the other patients' injuries in the sense of likelihood ratio orders. Nevertheless, the case where timecritical patients have longer service times appears to be more interesting and realistic, and hence we devoted a significant portion of the paper to identifying optimal or near-optimal policies for this case.

When time-critical jobs have stochastically longer service times, the optimal policy is not easy to characterize except for certain cases. For example, when service and lifetimes are exponentially distributed and the jobs can be categorized into two classes based on their mean service time and lifetimes, we were able to identify conditions under which it is always optimal to give priority to faster jobs, even when they are less time critical. For cases where we cannot characterize the optimal policy, we developed two state-dependent heuristic policies and compared them with two benchmark policies (that are not state-dependent) by means of a numerical study. From our numerical experiments, we gained several important insights. When the job abandonment rates are small compared to the service rates, as one would expect, all policies perform reasonably well (with one of the state-independent policies providing the best performance). On the other hand, when jobs abandon the system at a faster rate, the state-independent policies perform very poorly, and hence it is extremely important in this case to employ state-dependent policies such as those proposed in this paper. For the patient triage problem, these observations imply that when a major emergency event causes injuries that need to be taken care of very quickly, then it is crucial for a triage policy to take into account the number of patients who need help. Especially, for worst-case scenarios, where the event causes a large number of patients who need help immediately, our state-dependent heuristics appear to provide substantially better performance than the state-independent policies and have the potential to achieve the greatest good for the greatest number of people.

\section{Acknowledgement}

The work of the first author was supported by the National Science Foundation under Grant No. CMMI-0715020. The work of the second author was supported by the National Science Foundation under Grant No. CMMI-0620737. 


\section{References}

[1] Arnold, J. L., M.-C. Tsai, P. Halpern, H. Smithline, E. Stok, and G. Ersoy, "Masscasualty, terrorist bombings: Epidemiological outcomes, resource utilization, and time course of emergency needs (Part I)," Prehospital and Disaster Medicine 18 (2004), No. $3,220-234$.

[2] Bae, J., S. Kim, and E. Y. Lee, "The virtual waiting time of M/G/1 queue with impatient customers," Queueing Systems 38 (2001), 485-494.

[3] Bhattacharya, P. P., and A. Ephremides, "Optimal scheduling with strict deadlines," IEEE Transactions on Automatic Control 34 (1989), No. 7, 721-728.

[4] Bhattacharya, P. P., and A. Ephremides, "Optimal allocation of a server between two queues with due times," IEEE Transactions on Automatic Control 36 (1991), No. 12, $1417-1423$.

[5] Boxma, O. J., and F. G. Forst, "Minimizing the expected weighted number of tardy jobs in stochastic flow shops," Operations Research Letters 5 (1986), No. 3, 119-126.

[6] Brandt, A., and M. Brandt, "Asymptotic results and a Markovian appproximation for the $\mathrm{M}(n) / \mathrm{M}(n) / s$ + GI system," Queueing Systems 41 (2002), 73-94.

[7] Brandt, A., and M. Brandt, "On the two-class M/M/1 system under preemptive resume and impatience of the prioritized customers," Queueing Systems 47 (2004), 147-168.

[8] Choi, B. D., B. Kim, and J. Chung, "M/M/1 queue with impatient customers of higher priority," Queueing Systems 38 (2001), 49-66.

[9] Coffman, E. G., L. Flatto, M. R. Garey, and R. R. Weber, "Minimizing expected makespan on uniform processor systems," Advances in Applied Probability 19 (1987), $177-201$.

[10] Doytchinov, B., J. Lehoczky, and S. Shreve, "Real-time queues in heavy traffic with earliest-deadline-first queue discipline," Annals of Applied Probability 11 (2001), No. 2, $332-378$.

[11] Emmons, H., and M. Pinedo, "Scheduling stochastic jobs with due dates on parallel machines," European Journal of Operational Research 47 (1990), No. 1, 49-55.

[12] Frykberg, E. R., "Medical management of disasters and mass casualties from terrorist bombings: How can we cope?" The Journal of Trauma 53 (2002), No. 2, 201-212. 
[13] Glazebrook, K. D., "Stochastic scheduling with due dates," International Journal of Systems Science 14 (1983), 1259-1271.

[14] Glazebrook, K. D., P. S. Ansell, R. T. Dunn, and R. R. Lumley, "On the optimal allocation of service to impatient tasks," Journal of Applied Probability 41 (2004), No. $1,51-72$.

[15] Hougaard, P., Analysis of Multivariate Survival Data, Springer-Verlag, New York, NY, 2000.

[16] Jang, W., and C. M. Klein, "Minimizing the expected number of tardy jobs when processing times are normally distributed," Operations Research Letters 30 (2002), 100106.

[17] Jiang, Z., T. G. Lewis, and J.-Y. Colin, "Scheduling hard real-time constrained periodic tasks on multiple processors," Journal of Systems and Software 19 (1996), 102-118.

[18] Levi, L., M. Michaelson, H. Admi, D. Bregman, and R. Bar-Nahor, "National strategy for mass casualty situations and its effects on the hospital," Prehospital and Disaster Medicine 17 (2003), No. 1, 12-17.

[19] Nocera, A., and A. Garner, "An Australian Mass Casualty Incident triage system for the future based upon triage mistakes of the past: The Homebush Triage Standard," Australian and New Zealand Journal of Surgery 69 (1999), 603-608.

[20] Panwar, S. S., D. Towsley, and J. K. Wolf, "Optimal scheduling policies for a class of queues with customer deadlines to the beginning of service," Journal of the Association for Computing Machinery 35 (1988), No. 4, 832-844.

[21] Peleg, K., L. Aharonson-Daniel, M. Michael, S. C. Shapira, and the Israel Trauma Group, "Patterns of injury in hospitalized terrorist victims," American Journal of Emergency Medicine 21 (2003), No. 4, 258-262.

[22] Pinedo, M., "Stochastic Scheduling with release dates and due dates," Operations Research 31 (1983), No. 3, 559-572.

[23] Righter, R. "Scheduling," Stochastic Orders, ed. by M. Shaked and J. G. Shanthikumar. New York: Academic Press (1994), 381-432.

[24] Righter, R., "Job scheduling to minimize weighted flowtime on uniform processors," Systems and Control Letters 10 (1988), 211-216. 
[25] Righter, R., "Expulsion and scheduling control for multiclass queues with heterogenous servers," Queueing Systems 34 (2000), 289-300.

[26] Rund, D. A., and Rausch, T. S., Triage, The C. V. Mosby Company, St. Louis, MO, 1981.

[27] Van Mieghem, J., "Dynamic scheduling with convex delay costs: the generalized $c \mu$ rule," Annals of Applied Probability 5 (1995), No. 3, 808-833.

[28] Van Mieghem, J., "Due date scheduling: asymptotic optimality of generalized longest queue and generalized largest delay rules," Operations Research 51 (2003), No. 1, 113122.

[29] Ward, A. R., and P. W. Glynn, "A diffusion approximation for a Markovian queue with reneging," Queueing Systems 43 (2003), 103-128.

[30] Ward, A. R., and S. Kumar, "Asymptotically optimal admission control of a queue with impatient customers," submitted for publication, 2006.

[31] Weber, R. R., P. Varaiya, and J. Walrand, "Scheduling jobs with stochastically ordered processing times on parallel machines to minimize expected flow time," Journal of Applied Probability 23 (1986), 841-847.

[32] Weiss, G., and M. Pinedo, "Scheduling tasks with exponential service times on nonidentical processors to minimize various cost functions," Journal of Applied Probability 17 (1980), 187-202.

[33] Xu, S. H., "A duality approach to admission and scheduling control of queues," Queueing Systems 18 (1994), 273-300.

[34] Zhao, Z.-X., S. S. Panwar, and D. Towsley, "Queueing performance with impatient customers," Proceedings of IEEE INFOCOM'91 (1991), Vol. 1, 400-409.

\section{Appendix}

In this Appendix, we prove Proposition 7. We need the following lemma.

Lemma 3 Suppose that $\mu_{1}<\mu_{2}$.

(i) For all $x_{2} \geq 1$, we have

$$
V\left(0, x_{2} ; P_{2}\right)-V\left(1, x_{2}-1 ; P_{1}\right) \leq \frac{\left(x_{2}-1\right) r_{2}\left(\mu_{2}-\mu_{1}\right)}{\mu_{1}\left(\mu_{2}+\left(x_{2}-1\right) r_{2}\right)} .
$$


(ii) For all $x_{1} \geq 1$, we have

$$
V\left(x_{1}-1,1 ; P_{2}\right)-V\left(x_{1}, 0 ; P_{1}\right) \leq \frac{\left(x_{1}-1\right) r_{1}\left(\mu_{2}-\mu_{1}\right)}{\mu_{2}\left(\mu_{1}+\left(x_{1}-1\right) r_{1}\right)} .
$$

Proof of Lemma 3: (i) We prove the result by induction on $x_{2}$. Since $V\left(0,1 ; P_{2}\right)-$ $V\left(1,0 ; P_{1}\right)=0$, the result holds trivially for $x_{2}=1$. Next, suppose that the result holds for $x_{2}-1$. Then, we have

$$
\begin{aligned}
V( & \left.0, x_{2} ; P_{2}\right)-V\left(1, x_{2}-1 ; P_{1}\right) \\
= & \frac{\left(x_{2}-1\right) r_{2}\left(\mu_{2}-\mu_{1}\right)}{\left(\mu_{1}+\left(x_{2}-1\right) r_{2}\right)\left(\mu_{2}+\left(x_{2}-1\right) r_{2}\right)}+\frac{\left(x_{2}-1\right) r_{2}}{\mu_{1}+\left(x_{2}-1\right) r_{2}}\left(V\left(0, x_{2}-1 ; P_{2}\right)-V\left(1, x_{2}-2 ; P_{1}\right)\right) \\
\leq & \frac{\left(x_{2}-1\right) r_{2}\left(\mu_{2}-\mu_{1}\right)}{\left(\mu_{1}+\left(x_{2}-1\right) r_{2}\right)\left(\mu_{2}+\left(x_{2}-1\right) r_{2}\right)}+\frac{\left(x_{2}-1\right)\left(x_{2}-2\right) r_{2}^{2}\left(\mu_{2}-\mu_{1}\right)}{\mu_{1}\left(\mu_{1}+\left(x_{2}-1\right) r_{2}\right)\left(\mu_{2}+\left(x_{2}-2\right) r_{2}\right)} \\
& (\text { by the inductive hypothesis }) \\
= & \frac{\left(x_{2}-1\right) r_{2}\left(\mu_{2}-\mu_{1}\right)}{\mu_{1}\left(\mu_{2}+\left(x_{2}-1\right) r_{2}\right)}\left\{\frac{\mu_{1}}{\mu_{1}+\left(x_{2}-1\right) r_{2}}+\frac{\left(x_{2}-2\right) r_{2}\left(\mu_{2}+\left(x_{2}-1\right) r_{2}\right)}{\left(\mu_{1}+\left(x_{2}-1\right) r_{2}\right)\left(\mu_{2}+\left(x_{2}-2\right) r_{2}\right)}\right\} \\
\leq & \frac{\left(x_{2}-1\right) r_{2}\left(\mu_{2}-\mu_{1}\right)}{\mu_{1}\left(\mu_{2}+\left(x_{2}-1\right) r_{2}\right)},
\end{aligned}
$$

where the last inequality follows from the fact that $\left(x_{2}-2\right) r_{2}\left(\mu_{2}+\left(x_{2}-1\right) r_{2}\right) \leq\left(x_{2}-\right.$ 1) $r_{2}\left(\mu_{2}+\left(x_{2}-2\right) r_{2}\right)$.

(ii) We prove the result by induction on $x_{1}$. Since $V\left(0,1 ; P_{2}\right)-V\left(1,0 ; P_{1}\right)=0$, the result holds trivially for $x_{1}=1$. Next, suppose that the result holds for $x_{1}-1$. Then, we have

$$
\begin{aligned}
V & \left.x_{1}-1,1 ; P_{2}\right)-V\left(x_{1}, 0 ; P_{1}\right) \\
= & \frac{\left(x_{1}-1\right) r_{1}\left(\mu_{2}-\mu_{1}\right)}{\left(\mu_{1}+\left(x_{1}-1\right) r_{1}\right)\left(\mu_{2}+\left(x_{1}-1\right) r_{1}\right)}+\frac{\left(x_{1}-1\right) r_{1}}{\mu_{2}+\left(x_{1}-1\right) r_{1}}\left(V\left(x_{1}-2,1 ; P_{2}\right)-V\left(x_{1}-1,0 ; P_{1}\right)\right) \\
\leq & \frac{\left(x_{1}-1\right) r_{1}\left(\mu_{2}-\mu_{1}\right)}{\left(\mu_{1}+\left(x_{1}-1\right) r_{1}\right)\left(\mu_{2}+\left(x_{1}-1\right) r_{1}\right)}+\frac{\left(x_{1}-1\right)\left(x_{1}-2\right) r_{1}^{2}\left(\mu_{2}-\mu_{1}\right)}{\mu_{2}\left(\mu_{2}+\left(x_{1}-1\right) r_{1}\right)\left(\mu_{1}+\left(x_{1}-2\right) r_{1}\right)} \\
& \quad(\text { by the inductive hypothesis }) \\
\leq & \frac{\left(x_{1}-1\right) r_{1}\left(\mu_{2}-\mu_{1}\right)}{\mu_{2}\left(\mu_{1}+\left(x_{1}-1\right) r_{1}\right)} .
\end{aligned}
$$

Proof of Proposition 7: (i) We prove the result by induction on $x_{2}$. For $x_{2}=1$, we have $V\left(1,1 ; P_{2}\right)-V\left(1,1 ; P_{1}\right)=\left(r_{2} \mu_{2}-r_{1} \mu_{1}\right) /\left(\mu_{1}+r_{2}\right)\left(\mu_{2}+r_{1}\right) \geq 0$. Next, suppose that 
$V\left(1, x_{2}-1 ; P_{2}\right) \geq V\left(1, x_{2}-1 ; P_{1}\right)$. Then, we have

$$
\begin{aligned}
V(1, & \left.x_{2} ; P_{2}\right)-V\left(1, x_{2} ; P_{1}\right) \\
= & \frac{r_{2} \mu_{2}-r_{1} \mu_{1}}{\left(\mu_{1}+x_{2} r_{2}\right)\left(\mu_{2}+r_{1}+\left(x_{2}-1\right) r_{2}\right)}+\frac{\left(x_{2}-1\right) r_{2}\left(\mu_{2}-\mu_{1}\right)}{\left(\mu_{1}+x_{2} r_{2}\right)\left(\mu_{2}+r_{1}+\left(x_{2}-1\right) r_{2}\right)} \\
& +\left(\frac{\mu_{1}}{\mu_{1}+x_{2} r_{2}}-\frac{r_{1}}{\mu_{2}+r_{1}+\left(x_{2}-1\right) r_{2}}\right)\left(V\left(1, x_{2}-1 ; P_{1}\right)-V\left(0, x_{2} ; P_{2}\right)\right) \\
& +\frac{\mu_{2}+\left(x_{2}-1\right) r_{2}}{\mu_{2}+r_{1}+\left(x_{2}-1\right) r_{2}}\left(V\left(1, x_{2}-1 ; P_{2}\right)-V\left(1, x_{2}-1 ; P_{1}\right)\right) \\
\geq & \frac{\left(x_{2}-1\right) r_{2}\left(\mu_{2}-\mu_{1}\right)}{\left(\mu_{1}+x_{2} r_{2}\right)\left(\mu_{2}+r_{1}+\left(x_{2}-1\right) r_{2}\right)} \\
& +\left(\frac{\mu_{1}}{\mu_{1}+x_{2} r_{2}}-\frac{r_{1}}{\mu_{2}+r_{1}+\left(x_{2}-1\right) r_{2}}\right)\left(V\left(1, x_{2}-1 ; P_{1}\right)-V\left(0, x_{2} ; P_{2}\right)\right) \\
= & \left(\left(x_{2}-1\right) r_{2}+\frac{x_{2} r_{1} r_{2}-\mu_{1}\left(\mu_{2}+\left(x_{2}-1\right) r_{2}\right)}{\mu_{2}-\mu_{1}}\left(V\left(0, x_{2} ; P_{2}\right)-V\left(1, x_{2}-1 ; P_{1}\right)\right)\right) \\
& \times \frac{\left(\mu_{2}-\mu_{1}\right)}{\left(\mu_{1}+x_{2} r_{2}\right)\left(\mu_{2}+r_{1}+\left(x_{2}-1\right) r_{2}\right)} \\
\geq & \left(\left(x_{2}-1\right) r_{2}-\frac{\mu_{1}\left(\mu_{2}+\left(x_{2}-1\right) r_{2}\right)}{\mu_{2}-\mu_{1}}\left(V\left(0, x_{2} ; P_{2}\right)-V\left(1, x_{2}-1 ; P_{1}\right)\right)\right) \\
& \times \frac{\left(\mu_{2}-\mu_{1}\right)}{\left(\mu_{1}+x_{2} r_{2}\right)\left(\mu_{2}+r_{1}+\left(x_{2}-1\right) r_{2}\right)},
\end{aligned}
$$

since $V\left(1, x_{2}-1 ; P_{1}\right) \leq V\left(0, x_{2} ; P_{2}\right)$ when $\mu_{1}<\mu_{2}$ by Lemma 2 . Now, the condition that $\mu_{1}<\mu_{2}$ and part (i) of Lemma 3 complete the proof. 
(ii) We prove the result by induction on $x_{1}$. The case with $x_{1}=1$ is already covered in part (i). Now, suppose that $V\left(x_{1}-1,1 ; P_{2}\right) \geq V\left(x_{1}-1,1 ; P_{1}\right)$. Then, we have

$$
\begin{aligned}
V\left(x_{1},\right. & \left.1 ; P_{2}\right)-V\left(x_{1}, 1 ; P_{1}\right) \\
= & \frac{r_{2} \mu_{2}-r_{1} \mu_{1}}{\left(\mu_{2}+x_{1} r_{1}\right)\left(\mu_{1}+\left(x_{1}-1\right) r_{1}+r_{2}\right)}+\frac{\left(x_{1}-1\right) r_{1}\left(\mu_{2}-\mu_{1}\right)}{\left(\mu_{2}+x_{1} r_{1}\right)\left(\mu_{1}+\left(x_{1}-1\right) r_{1}+r_{2}\right)} \\
& +\left(\frac{\mu_{2}}{\mu_{2}+x_{1} r_{1}}-\frac{r_{2}}{\mu_{1}+\left(x_{1}-1\right) r_{1}+r_{2}}\right)\left(V\left(x_{1}, 0 ; P_{1}\right)-V\left(x_{1}-1,1 ; P_{2}\right)\right) \\
& +\frac{\left(x_{1}-1\right) r_{1}}{\mu_{1}+\left(x_{1}-1\right) r_{1}+r_{2}}\left(V\left(x_{1}-1,1 ; P_{2}\right)-V\left(x_{1}-1,1 ; P_{1}\right)\right) \\
\geq & \frac{\left(x_{1}-1\right) r_{1}\left(\mu_{2}-\mu_{1}\right)}{\left(\mu_{2}+x_{1} r_{1}\right)\left(\mu_{1}+\left(x_{1}-1\right) r_{1}+r_{2}\right)} \\
& +\left(\frac{\mu_{2}}{\mu_{2}+x_{1} r_{1}}-\frac{r_{2}}{\mu_{1}+\left(x_{1}-1\right) r_{1}+r_{2}}\right)\left(V\left(x_{1}, 0 ; P_{1}\right)-V\left(x_{1}-1,1 ; P_{2}\right)\right) \\
= & \left(\left(x_{1}-1\right) r_{1}+\frac{x_{1} r_{1} r_{2}-\mu_{2}\left(\mu_{1}+\left(x_{1}-1\right) r_{1}\right)}{\mu_{2}-\mu_{1}}\left(V\left(x_{1}-1,1 ; P_{2}\right)-V\left(x_{1}, 0 ; P_{1}\right)\right)\right) \\
& \times \frac{\left(\mu_{2}-\mu_{1}\right)}{\left(\mu_{2}+x_{1} r_{1}\right)\left(\mu_{1}+\left(x_{1}-1\right) r_{1}+r_{2}\right)} \\
\geq & \left(\left(x_{1}-1\right) r_{1}-\frac{\mu_{2}\left(\mu_{1}+\left(x_{1}-1\right) r_{1}\right)}{\mu_{2}-\mu_{1}}\left(V\left(x_{1}-1,1 ; P_{2}\right)-V\left(x_{1}, 0 ; P_{1}\right)\right)\right) \\
& \times \frac{\left(\mu_{2}-\mu_{1}\right)}{\left(\mu_{2}+x_{1} r_{1}\right)\left(\mu_{1}+\left(x_{1}-1\right) r_{1}+r_{2}\right)},
\end{aligned}
$$

since $V\left(x_{1}-1,1 ; P_{2}\right) \geq V\left(x_{1}, 0 ; P_{1}\right)$ when $\mu_{1}<\mu_{2}$ by Lemma 2 . Now, the condition that $\mu_{1}<\mu_{2}$ and part (ii) of Lemma 3 complete the proof. 